\title{
Cosmic rulers
}

\author{
Fabian Schmidt ${ }^{1, *}$ and Donghui Jeong ${ }^{2, \dagger}$ \\ ${ }^{1}$ Theoretical Astrophysics, California Institute of Technology, Mail Code 350-17, Pasadena, California 91125, USA \\ ${ }^{2}$ Department of Physics and Astronomy, Johns Hopkins University, 3400 N. Charles St., Baltimore, Maryland 21210, USA
}

(Received 10 May 2012; published 15 October 2012)

\begin{abstract}
We derive general covariant expressions for the six independent observable modes of distortion of ideal standard rulers in a perturbed Friedmann-Robertson-Walker spacetime. Our expressions are gauge invariant and valid on the full sky. These six modes are most naturally classified in terms of their rotational properties on the sphere, yielding two scalars, two vector (spin-1), and two tensor (spin-2) components. One scalar corresponds to the magnification, while the spin-2 components correspond to the shear. The vector components allow for a polar/axial decomposition analogous to the $E / B$ decomposition for the shear. Scalar modes do not contribute to the axial $(B$-)vector, opening a new avenue to probing tensor modes. Our results apply, but are not limited to, the distortion of correlation functions (of the cosmic microwave background, 21-cm emission, or galaxies) as well as to weak lensing shear and magnification, all of which can be seen as methods relying on "standard rulers."
\end{abstract}

DOI: 10.1103/PhysRevD.86.083527

PACS numbers: 98.80.Jk, 98.65.-r, 98.65.Dx

\section{INTRODUCTION}

One of the primary goals of cosmology is to accurately measure the expansion history and the growth of structure in the Universe. Many of the cosmological probes used for this purpose can be classified as standard candles or standard rulers. The most obvious examples are type Ia supernovae and the baryon acoustic oscillation feature in galaxy correlation functions, which in an unperturbed FriedmannRobertson-Walker (FRW) Universe directly measure the geometry and expansion history of the Universe [1-4]. Beyond the background cosmology, cosmological perturbations affect the apparent scale of rulers, which can be used as a probe of structure in the Universe. In fact, from this point of view, standard rulers comprise a much larger set of observations: for example, galaxy redshift surveys measure the correlation function of galaxies, which is then compared with predictions based on a cosmological model; in other words, the correlation length of galaxies (or any characteristic scale in their correlation function) serves as a standard ruler. Weak lensing shear, measured using galaxy ellipticities, uses the fact that galaxies' sizes measured along fixed directions are on average equal. On the other hand, lensing magnification measurements rely on the fact that galaxies have a characteristic luminosity (standard candle) and/or size (standard ruler). Of course, in the latter three cases the "ruler" has a large amount of scatter, so that one might call it a "statistical ruler." Another example of this kind is lensing reconstruction on diffuse backgrounds such as the cosmic microwave background (CMB) or 21-cm emission from the dark ages [5-7]. In this approach one uses the intrinsic correlation pattern of the background, which is known statistically, to reconstruct the distortion from the observed pattern.

\footnotetext{
*fabians@caltech.edu

†djeong@pha.jhu.edu
}

There is a simple, unified description of these various cosmological probes: we observe photons from two different directions and redshifts, which correspond to a known physical scale (e.g., the comoving sound horizon at recombination, or the characteristic size of a galaxy). In this paper, we study in a general covariant setting which underlying properties of the spacetime can be measured with an ideal standard ruler, working to linear order in perturbations. Since we have six parameters to vary when scanning over photon arrival directions and redshifts, we can measure six degrees of freedom. These can be interpreted as the components of a metric (of Euclidean signature) mapping apparent coordinate distances into actual physical separations at the source. It is useful to further decompose these components into parts parallel to the line of sight (longitudinal), transverse, and mixed longitudinal-transverse parts. This is equivalent to a decomposition into scalars, vectors, and tensors in the two-dimensional subspace perpendicular to the photon 4-momentum and the observer's 4-velocity; i.e., we classify components in terms of their transformation properties under a rotation around the line of sight. Note that this is independent of the usual decomposition of metric perturbations on three-dimensional spatial hypersurfaces (i.e., in terms of the transformation of plane-wave metric perturbations under a rotation around the $k$-vector). We will denote the latter ("3-scalars" and so on) as $C, B_{i}, A_{i j}, \ldots$, and the former ("2-scalars" etc) as $\mathcal{C}, \mathcal{B}_{i}, \mathcal{A}_{i j}, \ldots$ The transverse components of the distortion, $\mathcal{A}_{i j}$, are perhaps best known. They correspond to the magnification (2-scalar) and shear (2-tensor). We show that in general one can also measure a longitudinal scalar and the two components of a vector on the sphere.

On scales much smaller than the horizon, an effective Newtonian description is sufficient, and this is what essentially all previous studies are based on. However, upcoming surveys will probe scales approaching the 
horizon, and an interpretation of these data sets can in principle be hampered by gauge ambiguities. In the case of the correlation of galaxy density contrast, this issue has attracted significant interest and has recently been resolved [8-11]. The unified treatment presented here resolves these issues for the wide set of cosmological observables mentioned above. More precisely, we obtain general coordinate-independent and gauge-invariant results for all observables, including the shear and magnification. The differential equation (optical equation) governing the magnification and shear was first derived in Ref. [12]. The magnification has been derived to first order in Ref. [13]. The shear has been derived to second order in the conformal-Newtonian (cN) gauge in Ref. [14], while Ref. [15] derived the shear for general backgrounds. To the best of our knowledge, the expression for the observable shear written in a general gauge is presented here for the first time. Further, all expressions are valid on the full sky. Our approach naturally includes the "metric shear" contribution introduced in Ref. [16], and we provide a straightforward physical interpretation of our result.

In addition, we show how the (2-)vector observable uncovered here can be decomposed into $E$ and $B$ modes in analogy with the shear, corresponding to polar and axial vector parts. As in the case of shear and CMB polarization, (3-)scalar perturbations do not contribute to the $B$ mode, while (3-)tensor perturbations contribute. This in principle offers another avenue to search for a stochastic gravitational wave background in large-scale structure, since no scalar perturbations contribute at linear order. However, a spectroscopic data set is likely necessary to reconstruct the vector component with an interesting signal-to-noise.

Apart from the linear treatment of metric perturbations, we make two further simplifying assumptions: first, we assume "small rulers" in the sense that rulers subtend a small apparent angle and redshift interval. Wide-angle effects are likely negligible for almost all applications (the large-scale baryon acoustic oscillation feature being perhaps the most important exception). A treatment of wide-angle effects necessarily involves a detailed model of the survey geometry, which is clearly beyond the scope of this paper. The second assumption is that any scatter or variation in the actual ("intrinsic") physical scale of the standard ruler is uncorrelated with large-scale perturbations. This will not hold true in general, since the physical systems used as rulers will be affected by their large-scale environment. One well-known example is the intrinsic alignment contribution to shear correlations [17]. Further examples include the distortion of correlation functions by large-scale tidal fields [18], or by a nonGaussian coupling of the density field to primordial degrees of freedom [19]. Since these "intrinsic effects" depend on the physics of the given ruler, we refrain from discussing them here, as they would distract from the generality of the rest of the results.
Finally, while we focus on general standard rulers here, the case of standard candles is directly related to our results. This is because the relation between angular diameter distance $D_{A}$ and luminosity distance $D_{L}$,

$$
D_{L}=(1+\tilde{z})^{2} D_{A},
$$

where $\tilde{z}$ is the observed redshift, holds in a general spacetime and for any source (this is a consequence of photon phasespace conservation). Thus, the magnification measured for standard candles is identical to the magnification for standard rulers which we will derive here. However, as discussed, standard rulers can measure five additional degrees of freedom not accessible to standard candles.

Our results are of immediate relevance to recent studies which consider the $B$ modes of the cosmic shear as a possible probe of an inflationary gravitational wave background [16,20,21], and to studies that propose to use the high-redshift $21-\mathrm{cm}$ emission for the same purpose $[22,23]$. In particular, our expressions can be used directly to construct optimal estimators on the full sky for searching for the imprint of gravitational waves in a threedimensional field such as the $21-\mathrm{cm}$ background. We also use many of the results derived here in two recent papers studying the impact of gravitational waves on the observed large-scale structure $[21,24]$.

The outline of the paper is as follows: we begin in Sec. II by introducing our metric convention and useful notation. The general expression for the mapping from apparent size to true physical size of the ruler is derived in Sec. III. We then decompose the contributions into longitudinal and transverse parts in Sec. IV. The following three sections deal with these different parts consecutively. We discuss and conclude in Sec. VIII. The appendices contain a large amount of additional reference material on multipole expansions of higher spin functions, the perturbed photon geodesic equation, and various test cases applied to our results.

\section{NOTATION} by

In a general gauge, the perturbed FRW metric is given

$$
\begin{aligned}
d s^{2}= & a^{2}(\eta)\left[-(1+2 A) d \eta^{2}-2 B_{i} d \eta d x^{i}\right. \\
& \left.+\left(\delta_{i j}+h_{i j}\right) d x^{i} d x^{j}\right],
\end{aligned}
$$

where we have assumed a spatially flat Universe (curvature can be included straightforwardly, at the expense of some extra notation). Here, $\eta$ denotes conformal time. Often, the spatial part is further expanded as

$$
h_{i j}=2 D \delta_{i j}+2 E_{i j} \text {, }
$$

where $E_{i j}$ is traceless. We shall also present the most interesting results in two popular gauges: the synchronous-comoving (sc) gauge, where $A=0=B_{i}$, so that 


$$
d s^{2}=a^{2}(\eta)\left[-d \eta^{2}+\left(\delta_{i j}+h_{i j}\right) d x^{i} d x^{j}\right] ;
$$

and the $\mathrm{cN}$ gauge, where $B_{i}=0=E_{i j}$. In the latter case, we denote $A=\Psi, D=\Phi$, conforming with standard notation, so that

$d s^{2}=a^{2}(\eta)\left[-(1+2 \Psi) d \eta^{2}+(1+2 \Phi) \delta_{i j} d x^{i} d x^{j}\right]$.

We also denote the background FRW metric (in the absence of perturbations) as $\bar{g}_{\mu \nu}=a^{2}(\eta) \eta_{\mu \nu}$.

It is useful to define projection operators parallel and perpendicular to the observed line-of-sight direction $\hat{n}^{i}$, so that for any spatial vector $X^{i}$ and tensor $E_{i j}$,

$$
\begin{aligned}
X_{\|} & \equiv \hat{n}_{i} X^{i}, & E_{\|} & \equiv \hat{n}_{i} \hat{n}_{j} E^{i j}, \\
X_{\perp}^{i} & \equiv \mathcal{P}^{i j} X_{j}, & \mathcal{P}^{i j} & \equiv \delta^{i j}-\hat{n}^{i} \hat{n}^{j} .
\end{aligned}
$$

Correspondingly, we define projected derivative operators,

$$
\partial_{\|} \equiv \hat{n}^{i} \partial_{i}, \quad \text { and } \quad \partial_{\perp}^{i} \equiv \mathcal{P}^{i j} \partial_{j} .
$$

Note that $\partial_{\perp}^{i}, \partial_{\|}$and $\partial_{\perp}^{i}, \partial_{\perp}^{j}$ do not commute. Further, we find

$$
\partial_{j} \hat{n}^{i}=\partial_{\perp j} \hat{n}^{i}=\frac{1}{\chi} \mathcal{P}_{j}{ }_{j},
$$

where $\chi$ is the norm of the position vector so that $\hat{n}^{i}=x^{i} / \chi$. Note that $\hat{n}^{i}$ and $\partial_{\|}$commute. More expressions can be found in Sec. II of Ref. [11].

Finally, it proves useful to decompose the quantities defined on the sphere, i.e., as a function of the unit lineof-sight vector $\hat{\mathbf{n}}$, in terms of their properties under a rotation around $\hat{\mathbf{n}}$. In particular, consider an orthonormal coordinate system $\left(\mathbf{e}_{1}, \mathbf{e}_{2}, \hat{\mathbf{n}}\right)$. If we rotate the coordinate system around $\hat{\mathbf{n}}$ by an angle $\psi$, so that $\mathbf{e}_{i} \rightarrow \mathbf{e}_{i}^{\prime}$, then the linear combinations $\mathbf{m}_{ \pm} \equiv\left(\mathbf{e}_{1} \mp i \mathbf{e}_{2}\right) / \sqrt{2}$ transform as

$$
\mathbf{m}_{ \pm} \rightarrow \mathbf{m}_{ \pm}^{\prime}=e^{ \pm i \psi} \mathbf{m}_{ \pm} .
$$

We say that a general function $f(\hat{\mathbf{n}})$ is spin- $s$ if it transforms under the same transformation as

$$
f(\hat{\mathbf{n}}) \rightarrow f(\hat{\mathbf{n}})^{\prime}=e^{i s \psi} f(\hat{\mathbf{n}}) .
$$

An ordinary scalar function on the sphere is clearly spin- 0 , while the unit vectors $\mathbf{m}_{ \pm}$defined above are spin \pm 1 fields. More details can be found in Appendix A. This decomposition is particularly useful for deriving multipole coefficients and angular power spectra. We also define

$$
X_{ \pm} \equiv m_{+}^{i} X_{i}, \quad E_{ \pm} \equiv m_{+}^{i} m_{\mp}^{j} E_{i j}
$$

for any 3-vector $X_{i}$ and 3-tensor $E_{i j}$.

For the quantitative results shown in Secs. V, VI, and VII, we assume a flat $\Lambda$ CDM cosmology with $h=0.72$, $\Omega_{m}=0.28$, a scalar spectral index $n_{s}=0.958$ and power spectrum normalization at $z=0$ of $\sigma_{8}=0.8$.

\section{STANDARD RULER}

In the absence of perturbations, photon geodesics are given by straight lines in conformal coordinates,

$$
\bar{x}^{\mu}(\chi)=\left(\eta_{0}-\chi, \hat{\mathbf{n}} \chi\right),
$$

where we have chosen the comoving distance $\chi$ as the affine parameter. Correspondingly, for a photon arriving from a direction $\hat{\mathbf{n}}$ with redshift $\tilde{z}$, we assign an "observed" position of emission $x^{\mu}$ given by

$$
\tilde{x}^{0}=\eta_{0}-\tilde{\chi}, \quad \tilde{x}^{i}=\hat{n}^{i} \tilde{\chi}, \quad \tilde{\chi} \equiv \bar{\chi}(\tilde{z}),
$$

where $\bar{\chi}(\tilde{z})$ denotes the comoving distance-redshift relation in the background Universe. The actual spacetime point of emission, denoted with $x^{\mu}$, is displaced from the observed positions by $\Delta x^{\mu}$ (see also Fig. 1),

$$
x^{\mu}=\tilde{x}^{\mu}+\Delta x^{\mu}(\hat{\mathbf{n}}, \tilde{z}) .
$$

Further, we will need the scale factor at emission. It is related to the inferred emission scale factor $\tilde{a} \equiv$ $1 /(1+\tilde{z})$ by

$$
\frac{a\left(x^{0}(\hat{\mathbf{n}}, \tilde{z})\right)}{\tilde{a}}=1+\Delta \ln a(\hat{\mathbf{n}}, \tilde{z}) .
$$

At first order, $\Delta \ln a=\Delta z /(1+\tilde{z})$, where $\Delta z$ is the difference between the observed redshift and the redshift that would be observed in an unperturbed Universe. Note that

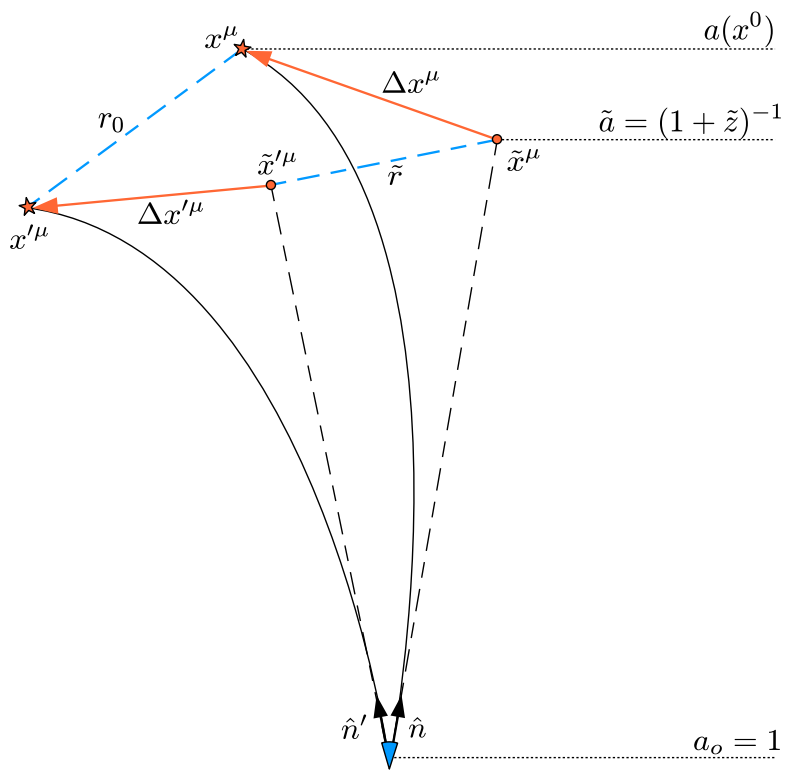

FIG. 1 (color online). Illustration of the apparent and actual standard ruler. Photons arrive out of the observed directions $\hat{\mathbf{n}}, \hat{\mathbf{n}}^{\prime}$ and with observed redshifts $\tilde{z}, \tilde{z}^{\prime}$. The apparent positions are indicated by $\tilde{x}^{\mu}, \tilde{x}^{\prime \mu}$, while the true positions are at $x^{\mu}, x^{\prime \mu}$, perturbed by the displacements $\Delta x^{\mu}, \Delta x^{\prime \mu}$ (whose magnitude is greatly exaggerated here). $\tilde{r}$ is the apparent size of the ruler, while $r_{0}$ is the true ruler. 
the latter quantity is gauge dependent. We will give explicit expressions for $\Delta \ln a$ and $\Delta x^{\mu}$ in Sec. IV.

The displacements $\Delta \ln a, \Delta x^{\mu}$ are not observable (they depend on which gauge, or frame, the spacetime perturbations are described in). In order to determine actual observables, we consider the case of a standard ruler. A standard ruler exists if we can identify two spacetime points which are separated by a fixed spacelike distance $r_{0}$. What we observe is the apparent size at which this ruler appears in a given direction $\hat{\mathbf{n}}$ and redshift $\tilde{z}$. Let $\hat{\mathbf{n}}, \tilde{z}$ and $\hat{\mathbf{n}}^{\prime}, \tilde{z}^{\prime}$ denote the observed coordinates of the "end points" of the ruler, and $\tilde{\mathbf{x}}$ and $\tilde{\mathbf{x}}^{\prime}$ the apparent spatial positions inferred through Eq. (13). The inferred physical separation is then given by

$$
\tilde{r}^{2}=\tilde{a}^{2} \delta_{i j}\left(\tilde{x}^{i}-\tilde{x}^{i i}\right)\left(\tilde{x}^{j}-\tilde{x}^{\prime j}\right),
$$

where $\tilde{a}=1 /(1+\tilde{z})$ is the observationally inferred scale factor at emission (Fig. 1).

We now have to carefully consider what the condition of a standard ruler in cosmology means. A useful, physically motivated definition is that it corresponds to a fixed spatial scale as measured by local observers which are comoving with the cosmic fluid; precisely, the spatial part of the fourvelocity $u^{\mu}$ of these observers is given by

$$
v^{i}=\frac{T_{0}^{i}}{\rho+p} .
$$

We are mostly interested in applications to the large-scale structure during matter domination; in this case, the cosmic fluid is simply matter (dark matter + baryons), and there is no ambiguity in this definition. In the sc gauge, Eq. (17) yields $v^{i}=0$. Further, for now we assume the ruler is fixed. We consider an evolving ruler in Sec. .

This definition can also be phrased as that the length of the ruler is defined on a surface of constant proper time of comoving observers. This proper time corresponds to the "local age" of the Universe. The separation of the two end points of the ruler, $x^{\mu}, x^{\prime \mu}$, projected onto this hypersurface should thus be equal to the fixed scale $r_{0}$ :

$$
\left[g_{\mu \nu}\left(x^{\alpha}\right)+u_{\mu}\left(x^{\alpha}\right) u_{\nu}\left(x^{\alpha}\right)\right]\left(x^{\mu}-x^{\prime \mu}\right)\left(x^{\nu}-x^{\prime \nu}\right)=r_{0}^{2},
$$

where $g_{\mu \nu}+u_{\mu} u_{\nu}$ is the metric projected perpendicular to $u_{\mu}$, the four-velocity of the comoving observers (note that $u_{\mu} u^{\mu}=-1$ ). Here and throughout, we will assume for simplicity that the ruler is "small"; i.e,. it subtends a small angle, and redshift interval $\left(\left|\tilde{z}-\tilde{z}^{\prime}\right| \ll \tilde{z}\right)$. This entails $\tilde{r} \ll \tilde{\chi}$, and that we can simply evaluate the metric and four-velocity at either end point (corrections involve higher powers of $\left.x^{\mu}-x^{\prime \mu}\right)$.

The four-velocity of comoving observers, whose spatial components are fixed by Eq. (17), is given by

$$
u^{\mu}=a^{-1}\left(1-A, v^{i}\right), \quad u_{\mu}=a\left(-1-A, v_{i}-B_{i}\right),
$$

where we consider $v^{i}$ to be first order (as the metric perturbations). In the following, we will assume sources to be comoving as well, i.e., to follow Eq. (19). It is straightforward to generalize the treatment to different source velocities. Using Eqs. (2) and (19), we have

$$
g_{\mu \nu}+u_{\mu} u_{\nu}=a^{2}\left(\begin{array}{cc}
0 & -v_{i} \\
-v_{i} & \delta_{i j}+h_{i j}
\end{array}\right) .
$$

With this, Eq. (18) yields

$$
\begin{aligned}
& -2 \tilde{a}^{2} v_{i}\left\{\delta \tilde{x}^{0} \delta \tilde{x}^{i}+\delta \tilde{x}^{0}\left[\Delta x^{i}-\Delta x^{\prime i}\right]+\delta \tilde{x}^{i}\left[\Delta x^{0}-\Delta x^{\prime 0}\right]\right\} \\
& \quad+g_{i j}\left(x^{\alpha}\right)\left\{\delta \tilde{x}^{i} \delta \tilde{x}^{j}+\delta \tilde{x}^{i}\left[\Delta x^{j}-\Delta x^{\prime j}\right]\right. \\
& \left.\quad+\left[\Delta x^{i}-\Delta x^{i}\right] \delta \tilde{x}^{j}\right\}=r_{0}^{2},
\end{aligned}
$$

where $\Delta x^{\mu}=\Delta x^{\mu}(\hat{\mathbf{n}}, \tilde{z}), \Delta x^{\prime \mu}=\Delta x^{\mu}\left(\hat{\mathbf{n}}^{\prime}, \tilde{z}^{\prime}\right)$, and the components of the apparent separation vector are

$$
\delta \tilde{x}^{\mu}=\tilde{x}^{\mu}-\tilde{x}^{\prime \mu} .
$$

In order to evaluate the spatial metric $g_{i j}\left(x^{\alpha}\right)$ at the location of the ruler, we use Eq. (15) to obtain at first order

$$
g_{i j}\left(x^{\alpha}\right)=\tilde{a}^{2}\left[(1+2 \Delta \ln a) \delta_{i j}+h_{i j}\right] .
$$

We now again make use of the small-ruler approximation, so that

$$
\Delta x^{i}-\Delta x^{\prime i} \simeq \delta \tilde{x}^{\alpha} \frac{\partial}{\partial \tilde{x}^{\alpha}} \Delta x^{i} .
$$

Like any vector, we can decompose the spatial part of the apparent separation $\delta \tilde{x}^{i}$ into parts parallel and transverse to the line of sight:

$\delta \tilde{x}_{\|} \equiv \hat{n}_{i} \delta \tilde{x}^{i}, \quad \delta \tilde{x}_{\perp}^{i} \equiv \mathcal{P}_{j}^{i} \delta \tilde{x}^{j}=\delta \tilde{x}^{i}-\hat{n}^{i} \delta \tilde{x}_{\|}$.

In the correlation function literature, $\delta \tilde{x}_{\|},\left|\delta \tilde{\mathbf{x}}_{\perp}\right|$ are sometimes referred to as $\pi$ and $\sigma$, respectively. Then,

$$
\delta \tilde{x}^{\alpha} \frac{\partial}{\partial \tilde{x}^{\alpha}}=\left(\delta \tilde{x}^{0} \partial_{\eta}+\delta \tilde{x}_{\|} \partial_{\|}\right)+\delta \tilde{x}_{\perp}^{i} \partial_{\perp i},
$$

where we have similarly defined $\partial_{\|}=\hat{n}^{i} \partial_{i}, \partial_{\perp i}=\mathcal{P}_{i}{ }^{j} \partial_{j}$. Since the observed coordinates $\tilde{x}^{\mu}$ by definition satisfy the light cone condition with respect to the unperturbed FRW metric, we have $\delta \tilde{x}^{0}=-\delta \tilde{x}_{\|}$in the small-angle approximation. Thus,

$$
\begin{aligned}
\delta \tilde{x}^{0} \partial_{\eta}+\delta \tilde{x}_{\|} \partial_{\|} & =\delta \tilde{x}_{\|}\left(\partial_{\|}-\partial_{\eta}\right)=\delta \tilde{x}_{\|} \frac{\partial}{\partial \tilde{\chi}} \\
& =\delta \tilde{x}_{\|} H(\tilde{z}) \frac{\partial}{\partial \tilde{z}},
\end{aligned}
$$

where $\partial / \partial \tilde{\chi}$ is the derivative with respect to the affine parameter at emission. We thus have

$$
\delta \tilde{x}^{\alpha} \frac{\partial}{\partial \tilde{x}^{\alpha}}=\delta \tilde{x}_{\|} \partial_{\tilde{\chi}}+\delta \tilde{x}_{\perp}^{i} \partial_{\perp i} .
$$


Working to first order in perturbations, we then obtain

$$
\begin{aligned}
r_{0}^{2}-\tilde{r}^{2}= & 2 \Delta \ln a \tilde{r}^{2}+\tilde{a}^{2} h_{i j} \delta \tilde{x}^{i} \delta \tilde{x}^{j} \\
& +2 \tilde{a}^{2}\left(v_{\|} \delta \tilde{x}_{\|}^{2}+v_{\perp i} \delta \tilde{x}_{\perp}^{i} \delta \tilde{x}_{\|}\right) \\
& +2 \tilde{a}^{2} \delta_{i j} \delta \tilde{x}^{i}\left(\delta \tilde{x}_{\|} \partial_{\tilde{x}}+\delta \tilde{x}_{\perp}^{k} \partial_{\perp k}\right) \Delta x^{j} .
\end{aligned}
$$

All terms are straightforward to interpret: there are the perturbations to the metric (both from the metric perturbation itself and the perturbation to the scale factor at emission); the contribution $\propto v$ from the projection from fixed- $\boldsymbol{\eta}$ to fixed-proper-time hypersurfaces; and the difference in the spatial displacements of the end points of the ruler.

\section{Evolving ruler}

So far, we have assumed that the physical scale of the ruler is fixed. This does not have to hold in general; for example, the baryon acoustic oscillation scale is fixed in terms of comoving coordinates. We now consider the case where the (mean) physical scale $r_{0}$ evolves over cosmic time. It is simplest to consider $r_{0}$ as a function of scale factor; one can easily convert to other variables such as conformal time using the relation with the scale factor in the background Universe.

Then, $r_{0}^{2}$ on the left-hand side of Eq. (29) is to be evaluated for the scale factor at emission:

$$
r_{0}^{2}\left[a\left(x^{0}\right)\right]=r_{0}^{2}(\tilde{a})\left[1+2 \frac{\partial \ln r_{0}}{\partial \ln a} \Delta \ln a\right] .
$$

Thus, if we compare the apparent size of the standard ruler to the true size at the apparent time of emission [of course assuming that we are able to predict $r_{0}(a)$ ], Eq. (29) becomes

$$
\begin{aligned}
r_{0}^{2}(\tilde{a})-\tilde{r}^{2}= & 2 \Delta \ln a\left[1-\frac{\partial \ln r_{0}}{\partial \ln a}\right] \tilde{r}^{2}+\tilde{a}^{2} h_{i j} \delta \tilde{x}^{i} \delta \tilde{x}^{j} \\
& +2 \tilde{a}^{2}\left(v_{\|} \delta \tilde{x}_{\|}^{2}+v_{\perp i} \delta \tilde{x}_{\perp}^{i} \delta \tilde{x}_{\|}\right) \\
& +2 \tilde{a}^{2} \delta_{i j} \delta \tilde{x}^{i}\left(\delta \tilde{x}_{\|} \partial_{\tilde{x}}+\delta \tilde{x}_{\perp}^{k} \partial_{\perp k}\right) \Delta x^{j} .
\end{aligned}
$$

Note that if $r_{0} \propto a$, i.e., if the ruler corresponds to a fixed comoving scale, the terms multiplying $\Delta \ln a$ cancel. This is as expected, since a perturbation to the scale factor at emission does not affect a fixed comoving scale.

\section{SCALAR-VECTOR-TENSOR DECOMPOSITION ON THE SKY}

It is useful to separate the contributions to Eq. (29) in terms of the observed longitudinal and transverse displacements. For some applications, only the transverse displacements are relevant. This is the case for diffuse backgrounds without redshift resolution, such as the $\mathrm{CMB}$ or the cosmic infrared background, and largely the case for photometric galaxy surveys. On the other hand, spectroscopic surveys and redshift-resolved backgrounds such as the 21-cm emission from high redshifts are able to measure the longitudinal displacements as well.

Noting that $\tilde{r}^{2}=\tilde{a}^{2}\left[\delta \tilde{x}_{\|}^{2}+\left(\delta \tilde{\mathbf{x}}_{\perp}\right)^{2}\right]$, and taking the square root of Eq. (31), we obtain the relative perturbation to the physical scale of the ruler as

$\frac{\tilde{r}-r_{0}}{\tilde{r}}=\mathcal{C} \frac{\left(\delta \tilde{x}_{\|}\right)^{2}}{\tilde{r}_{c}^{2}}+\mathcal{B}_{i} \frac{\delta \tilde{x}_{\|} \delta \tilde{x}_{\perp}^{i}}{\tilde{r}_{c}^{2}}+\mathcal{A}_{i j} \frac{\delta \tilde{x}_{\perp}^{i} \delta \tilde{x}_{\perp}^{j}}{\tilde{r}_{c}^{2}}$,

where we have defined $\tilde{r}_{c} \equiv \tilde{r} / \tilde{a}$ as the apparent comoving size of the ruler. The quantities multiplying $\mathcal{C}, \mathcal{B}_{i}, \mathcal{A}_{i j}$ are thus simply geometric factors. The coefficients are given by

$$
\begin{aligned}
\mathcal{C}= & -\Delta \ln a\left[1-\frac{\partial \ln r_{0}}{\partial \ln a}\right]-\frac{1}{2} h_{\|}-v_{\|}-\partial_{\tilde{\chi}} \Delta x_{\|} \\
\mathcal{B}_{i}= & -\mathcal{P}_{i}^{j} h_{j k} \hat{n}^{k}-v_{\perp i}-\hat{n}^{k} \partial_{\perp i} \Delta x_{k}-\partial_{\tilde{\chi}} \Delta x_{\perp i} \\
\mathcal{A}_{i j}= & -\Delta \ln a\left[1-\frac{\partial \ln r_{0}}{\partial \ln a}\right] \mathcal{P}_{i j}-\frac{1}{2} \mathcal{P}_{i}{ }^{k} \mathcal{P}_{j}{ }_{j} h_{k l} \\
& -\frac{1}{2}\left(\mathcal{P}_{j k} \partial_{\perp i}+\mathcal{P}_{i k} \partial_{\perp j}\right) \Delta x^{k},
\end{aligned}
$$

where $\Delta x_{\|}, \Delta x_{\perp}^{i}$ are the parallel and perpendicular components of the displacements $\Delta x^{i}$. Note that while we have assumed that the ruler is small (i.e., $\delta \tilde{x}^{i} \ll \tilde{\chi}$ ), the expressions for $\mathcal{C}, \mathcal{B}_{i}, \mathcal{A}_{i j}$ are valid on the full sky. Figure 2 illustrates the distortions induced by these components. Observationally, we have six free parameters (assuming accurate redshifts are available): the location of one point $\hat{\mathbf{n}}, \tilde{z}$, and the separation vector described by $\delta \tilde{x}^{i}$ (with $\delta \tilde{x}^{0}$

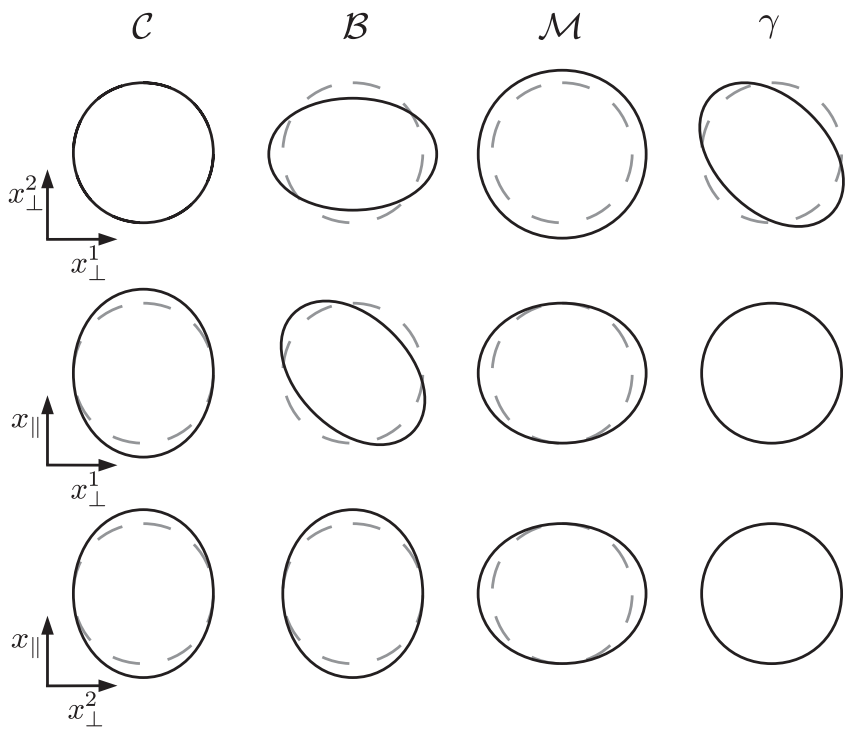

FIG. 2. Illustration of the distortion of standard rulers due to the longitudinal (2-)scalar $\mathcal{C}$, (2-)vector $\mathcal{B}$, and transverse components, magnification $\mathcal{M}$ and shear $\gamma$. The first row shows the projection onto the sky plane, while the second (third) row shows the projection onto the line-of-sight and $x_{\perp}^{1}\left(x_{\perp}^{2}\right)$ axis, respectively. In the cases of $\mathcal{B}$ and $\gamma$, we only show one of the two components. See also Fig. 3 in Ref. [12]. 
being fixed by the light cone condition). Using these, we can measure a (2-)scalar on the sphere, $\mathcal{C}$, a $2 \times 2$ symmetric matrix, $\mathcal{A}_{i j}$, and a two-component vector on the sphere, $\mathcal{B}_{i}$. As a symmetric matrix on the sphere, $\mathcal{A}_{i j}$ has a scalar component, given by the trace $\mathcal{M} \equiv \mathcal{P}^{i j} \mathcal{A}_{i j}$ (magnification), and two components of the traceless part which transform as spin-2 fields on the sphere [shear ${ }_{+2} \gamma$ as defined in Eq. (57) below]. These quantities are observable and gauge invariant, while any of the individual contributions in Eq. (33) are not in general. Note that we cannot measure any of the antisymmetric components, such as the rotation. This is because we have not assumed the existence of any preferred directions in the Universe. If there is a primary spin-1 or higher spin field, such as the polarization in case of the $\mathrm{CMB}$, then a rotation can be measured as it mixes the spin \pm 2 components (see, e.g., Ref. [25]). In the next sections we study these three terms in turn.

For reference, we now give the explicit expressions for the displacements $\Delta x^{i}$ and $\Delta \ln a$. They are defined such that $\Delta x^{i}=0=\Delta \ln a$ for a local source, i.e., for $\tilde{z} \approx 0$. The details of the derivation are presented in Appendix B. Separating into line-of-sight and transverse parts, we have

$$
\begin{aligned}
\Delta x_{\|}= & \int_{0}^{\tilde{\chi}} d \chi\left[A-B_{\|}-\frac{1}{2} h_{\|}\right]-\frac{1+\tilde{z}}{H(\tilde{z})} \Delta \ln a \\
\Delta x_{\perp}^{i}= & {\left[\frac{1}{2} \mathcal{P}^{i j}\left(h_{j k}\right)_{o} \hat{n}^{k}+B_{\perp o}^{i}-v_{\perp o}^{i}\right] \tilde{\chi} } \\
& +\int_{0}^{\tilde{\chi}} d \chi\left[-B_{\perp}^{i}-\mathcal{P}^{i j} h_{j k} \hat{n}^{k}+(\tilde{\chi}-\chi)\right. \\
& \left.\times\left\{-\partial_{\perp}^{i} A+\hat{n}^{k} \partial_{\perp}^{i} B_{k}+\frac{1}{2}\left(\partial_{\perp}^{i} h_{j k}\right) \hat{n}^{j} \hat{n}^{k}\right\}\right] \\
= & {\left[\frac{1}{2} \mathcal{P}^{i j}\left(h_{j k}\right)_{o} \hat{n}^{k}+B_{\perp o}^{i}-v_{\perp o}^{i}\right] \tilde{\chi} } \\
& -\int_{0}^{\tilde{\chi}} d \chi\left[\frac{\tilde{\chi}}{\chi}\left(B_{\perp}^{i}+\mathcal{P}^{i j} h_{j k} \hat{n}^{k}\right)\right. \\
& \left.+(\tilde{\chi}-\chi) \partial_{\perp}^{i}\left(A-B_{\|}-\frac{1}{2} h_{\|}\right)\right] .
\end{aligned}
$$

The perturbation to the scale factor at emission is given by

$$
\Delta \ln a=A_{o}-A+v_{\|}-v_{\| o}-\int_{0}^{\tilde{\chi}} d \chi\left[A-B_{\|}-\frac{1}{2} h_{\|}\right]^{\prime} .
$$

Here, a subscript $o$ indicates quantities evaluated at the observer, while primes denote derivatives with respect to $\eta$. Note the appearance of the scalar quantity $A-B_{\|}-\frac{1}{2} h_{\|}$ in Eqs. (34)-(37). This is the "lensing potential" $\Phi-\Psi$ in the cN gauge, written in the general gauge Eq. (2).

In particular, in the two popular gauges introduced in Sec. III, Eq. (37) becomes

$$
(\Delta \ln a)_{\mathrm{sc}}=\frac{1}{2} \int_{0}^{\tilde{\chi}} d \chi h_{\|}^{\prime}
$$

$(\Delta \ln a)_{\mathrm{cN}}=\Psi_{o}-\Psi+v_{\|}-v_{\| o}+\int_{0}^{\tilde{\chi}} d \chi\left[\Phi^{\prime}-\Psi^{\prime}\right]$.

The latter result clearly shows the "Sachs-Wolfe" gravitational redshift, "Doppler," and "integrated SachsWolfe" contributions.

\section{LONGITUDINAL SCALAR}

The longitudinal component can be simplified to become

$$
\begin{aligned}
\mathcal{C}= & -\Delta \ln a\left[1-H(\tilde{z}) \frac{\partial}{\partial \tilde{z}}\left(\frac{1+\tilde{z}}{H(\tilde{z})}\right)-\frac{\partial \ln r_{0}}{\partial \ln a}\right] \\
& -A-v_{\|}+B_{\|} \\
& +\frac{1+\tilde{z}}{H(\tilde{z})}\left(-\partial_{\|} A+\partial_{\|} v_{\|}+B_{\|}^{\prime}-v_{\|}^{\prime}+\frac{1}{2} h_{\|}^{\prime}\right) .
\end{aligned}
$$

The first line contains the contributions due to the fact that the scale factor at emission is perturbed from $1 /(1+\tilde{z})$ : first, the evolution of the physical standard ruler $r_{0}$ with scale factor, $1-\partial \ln r_{0} / \partial \ln a$, and second, the evolution of the distance-redshift relation. The second line contains the perturbations from the metric at the source location $(-A)$ and the projection from coordinate-time to proper-time hypersurfaces $\left(B_{\|}-v_{\|}\right)$. Finally, the contributions from the line-of-sight derivative of the line-of-sight displacements $[\propto(1+\tilde{z}) / H(\tilde{z})]$ are given in the third line. Note the term $\partial_{\|} v_{\|}$, which is the dominant term on small scales in the $\mathrm{cN}$ gauge. This term is also responsible for the leading-order redshift distortions [26]. Apart from the perturbation to the scale factor at emission, $\mathcal{C}$ does not involve any integral terms; this is expected since $\mathcal{C}$ is the only term remaining if the two lines of sight coincide $\left(\hat{\mathbf{n}}=\hat{\mathbf{n}}^{\prime}\right)$. In this case, the two rays share the same path from the closer of the two emission points, and no quantities integrated along the line of sight can contribute to the perturbation of the ruler.

Restricting to the sc and $\mathrm{cN}$ gauges, respectively, we obtain

$$
\begin{aligned}
(\mathcal{C})_{\mathrm{sc}}= & -(\Delta \ln a)_{\mathrm{sc}}\left[1-H(\tilde{z}) \frac{\partial}{\partial \tilde{z}}\left(\frac{1+\tilde{z}}{H(\tilde{z})}\right)-\frac{\partial \ln r_{0}}{\partial \ln a}\right] \\
& +\frac{1+\tilde{z}}{2 H(\tilde{z})} h_{\|}^{\prime} ; \\
(\mathcal{C})_{\mathrm{cN}}= & -(\Delta \ln a)_{\mathrm{cN}}\left[1-H(\tilde{z}) \frac{\partial}{\partial \tilde{z}}\left(\frac{1+\tilde{z}}{H(\tilde{z})}\right)-\frac{\partial \ln r_{0}}{\partial \ln a}\right] \\
& -\Psi-v_{\|}+\frac{1+\tilde{z}}{H(\tilde{z})}\left(-\partial_{\|} \Psi+\partial_{\|} v_{\|}-v_{\|}^{\prime}+\Phi^{\prime}\right) .
\end{aligned}
$$

Note that in the case of the sc-gauge expression, the redshift-space distortion term is included in the last term, through $h_{\|}^{\prime} / 2=D^{\prime}+\partial_{\|}^{2} E^{\prime}$. Figure 3 shows the angular power spectrum of $\mathcal{C}$ due to standard adiabatic scalar perturbations in a $\Lambda \mathrm{CDM}$ cosmology (the details of the 


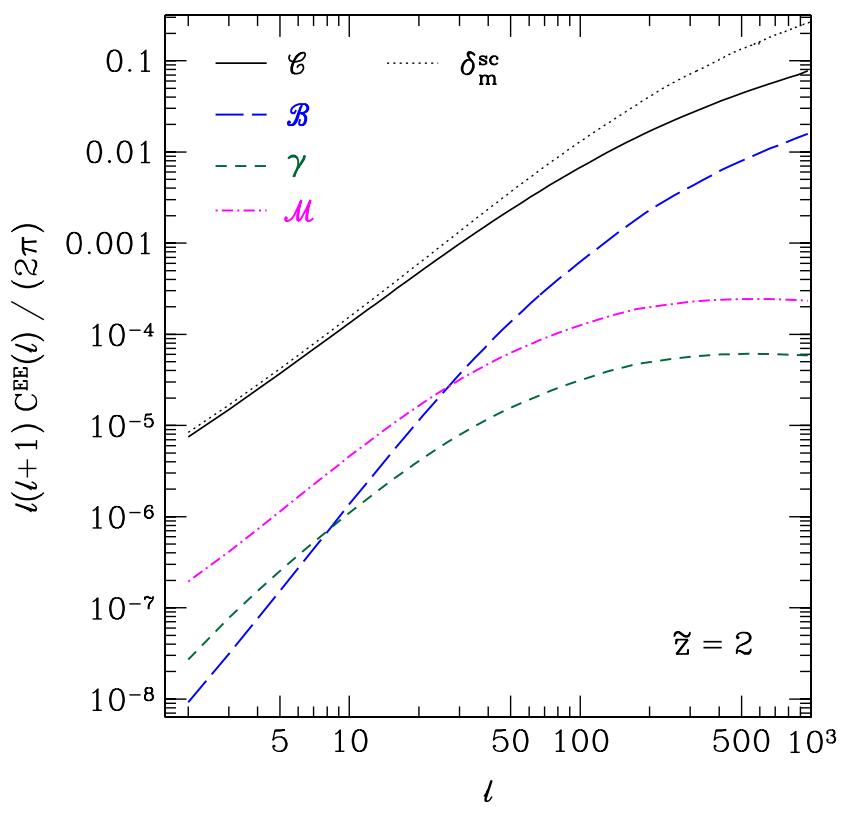

FIG. 3 (color online). Angular power spectra of the different standard ruler perturbations produced by a standard scaleinvariant power spectrum of curvature perturbations: $\mathcal{C}, E$ mode of $\mathcal{B}_{i}, E$ mode of the shear, and magnification $\mathcal{M}$. All quantities are calculated for a nonevolving ruler and a sharp source redshift of $\tilde{z}=2$. For comparison, the thin dotted line shows the angular power spectrum at $z=2$ of the matter density field in the sc gauge. Note that all quantities shown here, except for $\delta_{m}^{\mathrm{sc}}$, are gauge invariant and (in principle) observable.

calculation are given in Appendix E). Clearly, $\mathcal{C}$ is of the same order as the matter density contrast in the sc gauge on all scales. In particular, the velocity gradient term dominates over all other contributions. Due to the different dependence on the angle with the line of sight, the projection kernel of $\mathcal{C}$ is proportional to $\partial_{x}^{2} j_{l}(x)$, while that of $\delta_{m}^{\mathrm{sc}}$ is $\propto j_{l}(x)$. The former favors larger $x$ at a given $l$, and thus leads to a relative suppression as the slope of the matter power spectrum changes at $k \gtrsim 0.01 \mathrm{~h} / \mathrm{Mpc}$.

\section{VECTOR}

Next, we have the two-component vector

$$
\begin{aligned}
\mathcal{B}_{i} & =-\mathcal{P}_{i}{ }^{j} h_{j k} \hat{n}^{k}-v_{\perp i}-\partial_{\perp i} \Delta x_{\|}-\partial_{\tilde{\chi}} \Delta x_{\perp i}+\frac{\Delta x_{\perp i}}{\tilde{\chi}} \\
& =-v_{\perp i}+B_{\perp i}+\frac{1+\tilde{z}}{H(\tilde{z})} \partial_{\perp i} \Delta \ln a,
\end{aligned}
$$

where we have inserted projection operators for clarity (these are trivial since $\mathcal{B}_{i}$ is contracted with $\delta \tilde{x}_{\perp}^{i}$ ). As expected, this vector involves the transverse derivative of the line-of-sight displacement and the line-of-sight derivative of the transverse displacement. Note that these two quantities are not observable individually.

Using the spin \pm 1 unit vectors $\mathbf{m}_{ \pm}, \mathcal{B}_{i}$ can be decomposed into spin \pm 1 components:

$$
\begin{aligned}
\mathcal{B}_{i} & ={ }_{+1} \mathcal{B} m_{+}^{i}+{ }_{-1} \mathcal{B} m_{-}^{i}, \\
{ }_{ \pm 1} \mathcal{B} & \equiv m_{+}^{i} \mathcal{B}_{i}=-v_{ \pm}+B_{ \pm}+\frac{1+\tilde{z}}{H(\tilde{z})} \partial_{ \pm} \Delta \ln a,
\end{aligned}
$$

where we have used the notation of Eq. (11). Similar to before, we can specialize this general result to the sc and cN gauges:

$$
\begin{aligned}
& { }_{ \pm} \mathcal{B}()_{\mathrm{sc}}=\frac{1+\tilde{z}}{2 H(\tilde{z})} \int_{0}^{\tilde{\chi}} d \chi \frac{\chi}{\tilde{\chi}} \partial_{ \pm} h_{\|}^{\prime} \\
& \left({ }_{ \pm 1} \mathcal{B}\right)_{\mathrm{cN}}=-v_{ \pm}+\frac{1+\tilde{z}}{H(\tilde{z})} \partial_{ \pm} \Delta \ln a \\
& =-v_{ \pm}+\frac{1+\tilde{z}}{H(\tilde{z})}\left(-\partial_{ \pm} \Psi+\partial_{ \pm}\left[v_{\|}-v_{\| o}\right]\right. \\
& \left.+\int_{0}^{\tilde{\chi}} d \chi \frac{\chi}{\tilde{\chi}} \partial_{ \pm}\left(\Phi^{\prime}-\Psi^{\prime}\right)\right) .
\end{aligned}
$$

On small scales, the dominant contribution to $\mathcal{B}_{i}$ comes from the transverse derivative of the line-of-sight component of the velocity $\partial_{ \pm} v_{\|}$, which is of the same order as the tidal field.

Applying the spin-lowering operator $\bar{\delta}$ to ${ }_{1} \mathcal{B}$ (see Appendix A) yields a spin-0 quantity, which can be expanded in terms of the usual spherical harmonics. ${ }^{1}$ We then obtain the multipole coefficients of $\mathcal{B}$ as

$$
a_{l m}^{\mathcal{B}}(\tilde{z})=-\sqrt{\frac{(l-1) !}{(l+1) !}} \int d \Omega\left[\bar{\partial}_{1} \mathcal{B}(\hat{\mathbf{n}}, \tilde{z})\right] Y_{l m}^{*}(\hat{\mathbf{n}}) .
$$

An equivalent result is obtained for $\partial_{-1} \mathcal{B}$. In general, the multipole coefficients $a_{l m}^{\mathcal{B}}$ are complex, so that we can decompose them into real and imaginary parts,

$$
a_{l m}^{\mathcal{B}}=a_{l m}^{\mathcal{B E}}+i a_{l m}^{\mathcal{B B}} \text {. }
$$

One can easily show (Appendix A) that under a change of parity $a_{l m}^{\mathcal{B E}}$ transform as the spherical harmonic coefficients of a vector (parity odd), whereas $a_{l m}^{\mathcal{B B}}$, picking up an additional minus sign, transforms as those of a pseudovector (parity even). These thus correspond to the polar ("E") and axial (" $B$ ") parts of the vector $\mathcal{B}_{i}$.

As required by parity, scalar perturbations do not contribute to the axial part $a_{l m}^{\mathcal{B B}}$ (this is shown explicitly in Appendix D). Thus, a measurement of the vector component $\mathcal{B}_{i}$ of standard ruler distortions offers an additional possibility to probe tensor modes with large-scale structure, as tensor modes do contribute to $a_{l m}^{\mathcal{B B}}$ (Appendix D). Thus, in principle the axial component of $\mathcal{B}_{i}$ could be of similar interest for constraining tensor modes as weak lensing $B$ modes [21], though one likely requires accurate redshifts to measure $\mathcal{B}_{i}$ to sufficient accuracy. We leave a detailed investigation of this for future work.

\footnotetext{
${ }^{1}$ This is of course equivalent to expanding ${ }_{1} \mathcal{B}$ in terms of spin-1 spherical harmonics.
} 
The power spectrum of the $E$ mode of $\mathcal{B}$ due to standard scalar perturbations is shown in Sec. V through VII below (see Appendix E). While the dominant contribution to $\mathcal{C}$ is $\propto k_{\|}^{2} / k^{2} \delta_{m}^{\mathrm{sc}}(\mathbf{k}, \tilde{z})$ for a given Fourier mode of the matter density contrast in the sc gauge (Sec. V), the corresponding contribution to $\mathcal{B}$ is $\propto k_{\perp} k_{\|} / k^{2} \delta_{m}^{\mathrm{sc}}(\mathbf{k}, \tilde{z})$. Even though approximate scaling arguments suggest that $C_{\mathcal{C}}(l), C_{\mathcal{B}}^{E E}(l)$ should scale roughly equally with $l$, we see that $C_{\mathcal{B}}(l)$ scales faster with $l$ for $l \lesssim 500$. The reason is that the projection kernel for the $E$ mode of $\mathcal{B}\left[\propto\left(\partial_{x} j_{l}\right) / x\right]$ is relatively suppressed with respect to that of $\mathcal{C}\left(\propto \partial_{x}^{2} j_{l}\right)$ at large $x / l$. Since $l \leqq 500$ corresponds to a typical $k \lessgtr$ $10^{-2} h / \mathrm{Mpc}$ at the source redshift, where $P_{m}(k) \propto k$, larger $x / l$ are favored for progressively smaller $l$, leading to a more rapid decrease of $C_{\mathcal{B}}(l)$ towards smaller $l$. This suppression is thus fundamentally a consequence of the shape of the matter power spectrum.

\section{MAGNIFICATION AND SHEAR}

Finally, we have the purely transverse component,

$$
\begin{aligned}
\mathcal{A}_{i j}= & -\Delta \ln a\left[1-\frac{\partial \ln r_{0}}{\partial \ln a}\right] \mathcal{P}_{i j}-\frac{1}{2} \mathcal{P}_{i}{ }^{k} \mathcal{P}_{j}{ }_{j} h_{k l} \\
& -\partial_{\perp(i} \Delta x_{\perp j)}-\frac{1}{\tilde{\chi}} \Delta x_{\|} \mathcal{P}_{i j},
\end{aligned}
$$

where we have again inserted projection operators for clarity (note that $\mathcal{P}_{i j}$ serves as the identity matrix on the sphere). As a symmetric matrix on the sphere, $\mathcal{A}_{i j}$ has a scalar component, given by the trace $\mathcal{A}$, and two components of the traceless part which transform as spin-2 fields on the sphere. The trace corresponds to the change in area on the sky subtended by two perpendicular standard rulers. Thus, it is equal to the magnification $\mathcal{M}$ (see also Fig. 2). The two components of the traceless part correspond to the shear $\gamma$. If we choose a fixed coordinate system $\left(\mathbf{e}_{\theta}, \mathbf{e}_{\phi}, \hat{\mathbf{n}}\right)$, we can thus write

$$
\mathcal{A}_{i j}=\left(\begin{array}{ccc}
\mathcal{M} / 2+\gamma_{1} & \gamma_{2} & 0 \\
\gamma_{2} & \mathcal{M} / 2-\gamma_{1} & 0 \\
0 & 0 & 0
\end{array}\right) .
$$

Below, we will derive magnification and shear without reference to a fixed coordinate system.

\section{A. Magnification}

Taking the trace of Eq. (49) yields

$$
\begin{aligned}
\mathcal{M} & \equiv \mathcal{P}^{i j} \mathcal{A}_{i j} \\
& =-2 \Delta \ln a\left[1-\frac{\partial \ln r_{0}}{\partial \ln a}\right]-\frac{1}{2}\left(h^{i}{ }_{i}-h_{\|}\right)+2 \hat{\kappa}-\frac{2}{\tilde{\chi}} \Delta x_{\|} .
\end{aligned}
$$

The magnification is directly related to the fractional perturbations in distances (see Refs. $[27,28]$ ) through

$$
\frac{\Delta D_{L}}{D_{L}}=\frac{\Delta D_{A}}{D_{A}}=-\frac{1}{2} \mathcal{M},
$$

where the first equality for the luminosity distance follows from Eq. (1). The contributions to the magnification are straightforwardly interpreted as coming from the conversion of coordinate distance to physical scale at the source (from the perturbation to the scale factor $\Delta \ln a$ and the metric at the source projected perpendicular to the line of sight, $h_{i}^{i}-h_{\|}$); from the fact that the entire ruler is moved closer or farther away by $\Delta x_{\|}$; and finally from the coordinate convergence $\hat{\kappa}$ defined through

$$
\hat{\kappa}=-\frac{1}{2} \partial_{\perp i} \Delta x_{\perp}^{i} .
$$

This term dominates the other contributions to $\mathcal{M}$ on small scales. However, the coordinate convergence is a gauge-dependent quantity; see for example Appendix B2 in Ref. [11]. For the general metric Eq. (2) it is given by

$$
\begin{aligned}
\hat{\kappa}= & -\frac{1}{2}\left[\frac{1}{2}\left(\left(h^{i}{ }_{i}\right)_{o}-3\left(h_{\|}\right)_{o}\right)-2\left(B_{\|}-v_{\|}\right)_{o}\right] \\
& +\frac{1}{2} \int_{0}^{\tilde{\chi}} d \chi\left[\partial_{\perp}^{k} B_{k}-\frac{2}{\chi} B_{\|}+\left(\partial_{\perp}^{l} h_{l k}\right) \hat{n}^{k}\right. \\
& \left.+\frac{1}{\chi}\left(h^{i}{ }_{i}-3 h_{\|}\right)+(\tilde{\chi}-\chi) \frac{\chi}{\tilde{\chi}} \nabla_{\perp}^{2}\left\{A-B_{\|}-\frac{1}{2} h_{\|}\right\}\right] .
\end{aligned}
$$

In the $\mathrm{cN}$ gauge, it assumes its familiar form,

$(\hat{\kappa})_{\mathrm{cN}}=-v_{\| o}+\frac{1}{2} \int_{0}^{\tilde{\chi}} d \chi \frac{\chi}{\tilde{\chi}}(\tilde{\chi}-\chi) \nabla_{\perp}^{2}(\Psi-\Phi)$,

with an additional term $-v_{\| o}$ contributing to the dipole of $\hat{\kappa}$ only, which corresponds to the relativistic beaming effect at linear order. Explicit expressions for the magnification in different gauges are straightforward to obtain; however they become lengthy. Here we just show that we recover the result obtained in a different way in Jeong et al. [11] in the sc gauge. Using Eq. (3), $\left(h^{i}{ }_{i}-h_{\|}\right) / 2=2 D-E_{\|}$. This, and defining $\delta z \equiv$ $(\Delta \ln a)_{\mathrm{sc}}[$ Eq. (38)] yields

$$
(\mathcal{M})_{\mathrm{sc}}=-2\left(1-\frac{\partial \ln r_{0}}{\partial \ln a}\right) \delta z-2 D+E_{\|}+2 \hat{\kappa}-\frac{2}{\tilde{\chi}} \Delta x_{\|} .
$$

For a nonevolving standard ruler $\left(\partial \ln r_{0} / \partial \ln a=0\right)$, we thus recover the covariant magnification, $\mathcal{M}=\delta \mathcal{M}$, as derived in Ref. [11]. In general, the observable magnification $\mathcal{M}$ depends on the redshift evolution of the standard ruler considered; for example, galaxy sizes which can be used as a standard ruler to measure magnification [29] in general show a nontrivial redshift evolution. 


\section{B. Shear}

We now consider the traceless part of $\mathcal{A}_{i j}$, given by

$$
\begin{aligned}
\gamma_{i j}(\hat{\mathbf{n}}) & \equiv \mathcal{A}_{i j}-\frac{1}{2} \mathcal{P}_{i j} \mathcal{M} \\
& =-\frac{1}{2}\left(\mathcal{P}_{i}{ }^{k} \mathcal{P}_{j}{ }^{l}-\frac{1}{2} \mathcal{P}_{i j} \mathcal{P}^{k l}\right) h_{k l}-\partial_{\perp(i} \Delta x_{\perp j)}-\mathcal{P}_{i j} \hat{\kappa} .
\end{aligned}
$$

Here, the terms $\propto \mathcal{P}_{i j}$ in Eq. (49) drop out. The last two terms here are what commonly is regarded as the shear, i.e., the trace-free part of the transverse derivatives of the transverse displacements. The first term on the other hand is important to ensure a gauge-invariant result. This is the term referred to as metric shear in Ref. [16]. Its physical significance becomes clear when constructing the Fermi normal coordinates for the region containing the standard ruler.

Consider a region of spatial extent $R$, say centered on a given galaxy, with $R$ assumed to be much larger than the scale of individual galaxies. We can construct orthonormal Fermi normal coordinates [30,31] around the center of this region, which follows a timelike geodesic, by choosing the origin to be located at the center of the region at all times, and the time coordinate to be the proper time of this geodesic. The spacetime in these Fermi coordinates $\left(t_{F}, x_{F}^{i}\right)$ then becomes Minkowski, with corrections going as $x_{F}^{2} / R_{c}^{2}$ where $R_{c}$ is the curvature scale of the spacetime. Thus, as long as these corrections to the metric are negligible, there is no preferred direction in this frame, and the size of the standard ruler has to be (statistically) independent of the orientation. The most obvious example is galaxy shapes, which are used for cosmic shear measurements. In the Fermi frame, galaxy orientations are random. Note that the Fermi coordinates are uniquely determined up to three Euler angles. The statement that galaxy orientations are random in this frame is thus coordinate invariant.

As an example, consider the case where we have a purely spatial metric perturbation [cf. Eq. (2)] at a fixed time. We can then expand around the origin,

$$
h_{i j}(\mathbf{x})=h_{i j}(0)+h_{i j, k}(0) x^{k} .
$$

Higher-order terms are suppressed by $\left(x / R_{c}\right)^{2}$. Now, consider coordinates given by

$$
a^{-1} x_{F}^{i}=x^{i}+\frac{1}{2} h_{i j}(0) x^{j}+\frac{1}{4}\left[2 h_{i j, k}(0)-h_{j k, i}(0)\right] x^{j} x^{k} .
$$

In these coordinates, the metric becomes

$$
g_{\mu \nu}^{F}=\eta_{\mu \nu}+\mathcal{O}\left(x_{F}^{2}\right) .
$$

Thus, it is in terms of the coordinates $x_{F}^{i}$ that galaxies should be isotropically oriented on average, not in terms of the cosmological coordinates $x^{i}$. Correspondingly, in order to obtain the shear relative to the Fermi frame, we need to add the transformation Eq. (59) to the displacements $\Delta x^{i}$ :

$\Delta x^{i} \rightarrow \Delta x^{i}+\frac{1}{2} h_{i j}(0) x^{j}+\frac{1}{4}\left[2 h_{i j, k}(0)-h_{j k, i}(0)\right] x^{j} x^{k}$.

With these new displacements, the transverse derivative of the transverse displacement becomes

$\partial_{\perp(i} \Delta x_{\perp j)} \rightarrow \partial_{\perp(i} \Delta x_{\perp j)}+\frac{1}{2} \mathcal{P}_{i}{ }^{k} \mathcal{P}_{j}{ }_{j} h_{k l}+\mathcal{O}\left(h_{i j, k} x^{k}\right)$,

where the last term is suppressed by the size of the ruler over the wavelength of the metric perturbation, and is thus negligible in the small-ruler approximation. We see that Eq. (62) agrees exactly with the result derived above, Eq. (57) [after subtracting the trace of Eq. (62)]. In other words, the shear derived in the standard ruler formalism (Sec. III) is equivalent to the statement that the ruler is isotropic in its Fermi frame, the additional term coming from the transformation from global coordinates to the local Fermi coordinates. This additional term was introduced in Ref. [16] as metric shear, with a similar motivation as given here. In our case, this term is naturally included in the standard ruler formalism.

$\gamma_{i j}$ is a symmetric trace-free tensor on the sphere, and can thus be decomposed into spin \pm 2 components (in analogy to the polarization of the $\mathrm{CMB}$ ). Following Appendix A (see also Ref. [32]) we can write $\gamma_{i j}$ as

$\gamma_{i j}={ }_{2} \gamma m_{+}^{i} m_{+}^{j}+{ }_{-2} \gamma m_{-}^{i} m_{-}^{j}, \quad{ }_{ \pm 2} \gamma=m_{+}^{i} m_{+}^{j} \gamma_{i j}$,

where ${ }_{ \pm 2} \gamma$ are spin \pm 2 functions on the sphere (in analogy to the combination of Stokes parameters $Q \pm i U)$. We obtain for the shear components

$$
\begin{aligned}
{ }_{ \pm 2} \gamma= & -\frac{1}{2} h_{ \pm}-m_{+}^{i} m_{\mp}^{j} \partial_{\perp i} \Delta x_{\perp j} \\
= & -\frac{1}{2} h_{ \pm}-\frac{1}{2}\left(h_{ \pm}\right)_{o}-\int_{0}^{\tilde{\chi}} d \chi\left[( 1 - 2 \frac { \chi } { \tilde { \chi } } ) \left[m_{+}^{k} \partial_{ \pm} B_{k}\right.\right. \\
& \left.+\left(\partial_{ \pm} h_{l k}\right) m_{\mp}^{l} \hat{n}^{k}\right]-\frac{1}{\tilde{\chi}} h_{ \pm}+(\tilde{\chi}-\chi) \frac{\chi}{\tilde{\chi}} \\
& \times\left\{-m_{+}^{i} m_{\mp}^{j} \partial_{i} \partial_{j} A+\hat{n}^{k} m_{+}^{i} m_{\mp}^{j} \partial_{i} \partial_{j} B_{k}\right. \\
& \left.\left.+\frac{1}{2} m_{+}^{i} m_{+}^{j}\left(\partial_{i} \partial_{j} h_{k l}\right) \hat{n}^{k} \hat{n}^{l}\right\}\right]
\end{aligned}
$$

Equation (64) is valid in any gauge. We can now specialize to the $\mathrm{sc}$ and $\mathrm{cN}$ gauges:

$$
\begin{aligned}
\left({ }_{ \pm 2} \gamma\right)_{\mathrm{sc}}= & -\frac{1}{2} h_{ \pm}-\frac{1}{2}\left(h_{ \pm}\right)_{o}-\int_{0}^{\tilde{\chi}} d \chi\left[\left(1-2 \frac{\chi}{\tilde{\chi}}\right)\right. \\
& \times\left(\partial_{ \pm} h_{k l}\right) m_{+}^{k} \hat{n}^{l}-\frac{1}{\tilde{\chi}} h_{ \pm}+(\tilde{\chi}-\chi) \frac{\chi}{\tilde{\chi}} \frac{1}{2} \\
& \left.\times\left(m_{+}^{i} m_{+}^{j} \partial_{i} \partial_{j} h_{l k}\right) \hat{n}^{l} \hat{n}^{k}\right]
\end{aligned}
$$


$\left.{ }_{ \pm 2} \gamma\right)_{\mathrm{cN}}=\int_{0}^{\tilde{\chi}} d \chi(\tilde{\chi}-\chi) \frac{\chi}{\tilde{\chi}} m_{+}^{i} m_{\mp}^{j} \partial_{i} \partial_{j}(\Psi-\Phi)$.

In case of the $\mathrm{cN}$ gauge, we have used that $h_{i j}=2 \Phi \delta_{i j}$, and thus $h_{ \pm}=0$. We see that Eq. (66) recovers the "standard" result; in other words, there are no additional relativistic corrections to the shear in this gauge. This is not surprising following our arguments above: in the $\mathrm{cN}$ gauge, the transformation Eq. (59) from global coordinates to the local Fermi frame is isotropic since $h_{i j}=2 \Phi \delta_{i j}$. Thus, it does not contribute to the shear. Note however that only scalar perturbations are included in this gauge; when considering vector or tensor perturbations, one has to use a different gauge, for example the sc gauge (see Ref. [21] for a study of tensor perturbations). Thus, Eqs. (64) and (65) are important new results.

In Appendix C, we apply several test cases to the shear in the sc gauge, Eq. (65), in order to verify that it is gauge invariant and correctly reproduces known results. In particular, we consider a Bianchi I cosmology which induces a shear due to the anisotropic angular diameter distance. We also show that Eq. (65), when restricted to scalar perturbations, does not produce $B$-mode shear.

Figure 3 shows the angular power spectrum of shear and magnification due to scalar perturbations for a sharp source redshift $\tilde{z}=2$ (see Appendix E). For $l \geqq 10$, the results follow the familiar relation $C_{\mathcal{M}}(l)=4 C_{\gamma}^{E E}(l)$, valid when all relativistic corrections to the magnification become irrelevant so that $\mathcal{M} \simeq 2 \hat{\kappa}$. These corrections slightly increase the magnification for small $l$. We also see that $\gamma$ and $\mathcal{M}$ are suppressed with respect to $\mathcal{C}$ and $\mathcal{B}$ (on smaller scales), at least when the latter are evaluated for a sharp source redshift. This is a well-known consequence of the projection with the broad lensing kernel, leading to a cancellation of modes that are not purely transverse (see e.g., Ref. [33]).

\section{DISCUSSION}

Over the past decade, cosmology has benefited from a vast increase in the available data, which have been exploited through a broad variety of methods to constrain the history of structure in the Universe. Clearly, this calls for a rigorous investigation of what quantities precisely are observable in the relativistic setting. Some observables have been investigated previously, most notably the number density of tracers and the magnification. Here, we have presented a unified relativistic analysis of "standard rulers," where a standard ruler simply means there is an underlying physical scale which we compare the observations to. This treatment applies to lensing measurements through galaxy ellipticities, sizes and fluxes, or through standard candles, to distortions of cosmological correlation functions, and to lensing of diffuse backgrounds.

We show that in this framework, for ideal measurements, one can measure six degrees of freedom: a scalar corresponding to purely line-of-sight effects; a vector (on the sphere) which corresponds to mixed transverse/line-ofsight effects; and a symmetric transverse tensor on the sphere which comprises the shear and magnification. We obtain general, gauge-invariant expressions for the six observable degrees of freedom, valid on the full sky. These constitute the main result of the paper and are given in Eqs. (40), (44), (51), and (64). The vector component and the shear admit a decomposition into $E / B$ modes. The $B$ modes are free of all scalar contributions (including lensing as well as redshift-space distortions) at the linear level, making them ideal probes to look for tensor perturbations. As an application of our results, we study the shear induced by tensor modes (gravitational waves) in Ref. [21].

The logical next step is to construct estimators for these degrees of freedom, based on measurements of the density field of tracers (such as galaxies, the Lyman- $\alpha$ forest, $21-\mathrm{cm}$ emission, and so on). We will leave this for future work.

\section{ACKNOWLEDGMENTS}

We would like to thank Yanbei Chen, Scott Dodelson, Olivier Doré, Sam Gralla, Chris Hirata, Wayne Hu, Bhuvnesh Jain, Marc Kamionkowski, Eiichiro Komatsu, David Nichols, and Samaya Nissanke for helpful discussions. F. S. thanks Masahiro Takada and the Kavli-IPMU, University of Tokyo, for hospitality. F. S. is supported by the Gordon and Betty Moore Foundation at Caltech.

\section{APPENDIX A: SPHERICAL HARMONIC DECOMPOSITION OF SPIN-s FUNCTIONS}

Here we outline our notation and useful results on the spherical harmonic decomposition of tensors on the sphere. Throughout, latin indices $i, j, \ldots$ denote components with respect to Euclidean coordinates, and are raised and lowered with $\delta_{i j}$. We follow standard convention; see Ref. [32]. In particular, we do not include the CondonShortley phase in the spherical harmonics, so that $\left(Y_{l m}\right)^{*}=$ $Y_{l-m}$. Explicitly, in our convention the spherical harmonics are given by

$Y_{l m}(\theta, \phi)=\epsilon_{m} \sqrt{\frac{2 l+1}{4 \pi} \frac{(l-|m|) !}{(l+|m|) !}} P_{l}^{|m|}(\cos \theta) e^{i m \phi}$,

where $\epsilon_{m}$ is a phase factor defined as

$$
\boldsymbol{\epsilon}_{m}= \begin{cases}1, & m>0 \\ (-1)^{m}, & m \leq 0\end{cases}
$$

We can define spin \pm 1 unit basis vectors on the unit sphere

$m_{ \pm}^{i} \equiv \frac{1}{\sqrt{2}}\left(e_{\theta}^{i} \mp i e_{\phi}^{i}\right)=\frac{1}{\sqrt{2}}\left(\begin{array}{c}\cos \theta \cos \phi \pm i \sin \phi \\ \cos \theta \sin \phi \mp i \cos \phi \\ -\sin \theta\end{array}\right)$,

where $e_{\theta}, e_{\phi}$ are assumed orthonormal. $m_{ \pm}$transform as spin $\mp 1$ fields (see Sec. I). We have 


$$
\begin{aligned}
m_{ \pm}^{i} m_{ \pm i} & =0, & m_{ \pm}^{i} m_{\mp i} & =1, \\
m_{ \pm}^{i} \hat{n}_{i} & =0, & \mathcal{P}^{i j} m_{ \pm j} & =m_{ \pm}^{i} .
\end{aligned}
$$

Next, we define operators that raise and lower the spin $s$ of a function (or tensor component) ${ }_{s} f(\theta, \phi)$ through $[34,35]$

$$
\begin{aligned}
& {\mathrm{\jmath}_{s} f}=-\sin ^{s} \theta\left[\frac{\partial}{\partial \theta}+\frac{i}{\sin \theta} \frac{\partial}{\partial \phi}\right] \sin ^{-s} \theta_{s} f \\
& \bar{\partial}_{s} f=-\sin ^{-s} \theta\left[\frac{\partial}{\partial \theta}-\frac{i}{\sin \theta} \frac{\partial}{\partial \phi}\right] \sin ^{s} \theta_{s} f .
\end{aligned}
$$

A straightforward calculation using partial integration shows that

$$
\begin{aligned}
\int\left(\varlimsup_{s} f\right)_{s+1} g d \Omega= & \int\left(-\sin ^{s} \theta\right)\left(\left[\partial_{\theta}+i \sin ^{-1} \theta \partial_{\phi}\right]\right. \\
& \left.\times \sin ^{-s} \theta_{s} f\right)_{s+1} g \sin \theta d \theta d \phi \\
= & \int_{s} f \sin ^{-s-1} \theta\left(\left[\partial_{\theta}+i \sin ^{-1} \theta \partial_{\phi}\right]\right. \\
& \left.\times \sin ^{s+1} \theta_{s+1} g\right) \sin \theta d \theta d \phi \\
= & \int_{s} f\left(-\bar{\chi}^{*}{ }_{s+1} g\right) d \Omega
\end{aligned}
$$

in other words $-\bar{\delta}^{*}$ is the adjoint operator of $ð$ with respect to the standard measure on the sphere. In many cases, we will encounter functions given by ${ }_{s} f(\theta, \phi)=$ $e^{i m \phi} \tilde{f}(\mu)$, where $\mu=\cos \theta$. In this case, Eq. (A5) simplifies to

$\partial_{s} f=-\left(1-\mu^{2}\right)^{(1+s) / 2}\left[-\frac{\partial}{\partial \mu}-\frac{m}{1-\mu^{2}}\right]\left(1-\mu^{2}\right)^{-s / 2}{ }_{s} f$

$\bar{ळ}_{s} f=-\left(1-\mu^{2}\right)^{(1-s) / 2}\left[-\frac{\partial}{\partial \mu}+\frac{m}{1-\mu^{2}}\right]\left(1-\mu^{2}\right)^{s / 2} f$,

which applied twice straightforwardly yields

$$
\begin{aligned}
\bar{\partial}_{2}^{2} f(\mu, \phi) & =\left(-\frac{\partial}{\partial \mu}+\frac{m}{1-\mu^{2}}\right)^{2}\left[\left(1-\mu^{2}\right)_{2} f(\mu, \phi)\right] \\
\partial^{2}{ }_{-2} f(\mu, \phi) & =\left(-\frac{\partial}{\partial \mu}-\frac{m}{1-\mu^{2}}\right)^{2}\left[\left(1-\mu^{2}\right)_{-2} f(\mu, \phi)\right] .
\end{aligned}
$$

We can use the spin-raising and -lowering operators to define spin-weighted spherical harmonics through

$$
{ }_{s} Y_{l m}=\sqrt{\frac{(l-|s|) !}{(l+|s|) !}} \begin{cases}\partial^{s} Y_{l m}, & s \geq 0 \\ (-1)^{s} \bar{\partial}^{|s|} Y_{l m}, & s<0 .\end{cases}
$$

Note again that our spherical harmonics are defined such that $\left[Y_{l m}\right]^{*}=Y_{l-m}$. Equation (A5) together with Eq. (A9) then yield $\left[{ }_{s} Y_{l m}\right]^{*}=(-1)^{s}{ }_{-s} Y_{l-m}$. For $s= \pm 2$, this is equivalent to the definition used in Refs. [32,36]:

$$
{ }_{ \pm 2} Y_{l m}=2 \sqrt{\frac{(l-2) !}{(l+2) !}} m_{\mp}^{i} m_{\mp}^{j} \nabla_{i} \nabla_{j} Y_{l m}
$$

The spin-weighted spherical harmonics defined in Eq. (A9) form an orthonormal basis for spin-s functions. Using that $\partial^{\dagger}=-\bar{\partial}^{*}$, the orthonormality implies

$$
\bar{\partial}^{s} ð^{s} Y_{l m}=ð^{s} \bar{\partial}^{s} Y_{l m}=(-1)^{s} \frac{(l+|s|) !}{(l-|s|) !} Y_{l m} .
$$

Returning to the decomposition of a general spin $\pm s$ field (with $s>0$ ), we can express the components as

$$
{ }_{ \pm s} A(\hat{\mathbf{n}})=\sum_{l m} a_{l m \pm s}^{A} Y_{l m}(\hat{\mathbf{n}}) .
$$

Acting with the spin-lowering operator on ${ }_{+s} A$ and vice versa then yields

$$
\begin{aligned}
\bar{\partial}_{s}^{s} A(\hat{\mathbf{n}}) & =\sum_{l m} a_{l m}^{A} \sqrt{\frac{(l-s) !}{(l+s) !}} \bar{\partial}^{s} \partial^{s} Y_{l m}(\hat{\mathbf{n}}) \\
& =\sum_{l m} a_{l m}^{A}(-1)^{s} \sqrt{\frac{(l+s) !}{(l-s) !}} Y_{l m}(\hat{\mathbf{n}}) \\
\partial^{s}{ }_{-s} A(\hat{\mathbf{n}}) & =\sum_{l m} a_{l m}^{A}(-1)^{s} \sqrt{\frac{(l-s) !}{(l+s) !}} \partial^{s} \bar{\partial}^{s} Y_{l m}(\hat{\mathbf{n}}) \\
& =\sum_{l m} a_{l m}^{A} \sqrt{\frac{(l+s) !}{(l-s) !}} Y_{l m}(\hat{\mathbf{n}}) .
\end{aligned}
$$

We thus have

$$
\begin{aligned}
a_{l m}^{A} & =\int_{{ }_{ \pm}} A(\hat{\mathbf{n}})\left[{ }_{ \pm s} Y_{l m}(\hat{\mathbf{n}})\right]^{*} d \Omega \\
& =\sqrt{\frac{(l-|s|) !}{(l+|s|) !}}(-1)^{s} \int\left[\bar{\partial}^{s}{ }_{+s} A(\hat{\mathbf{n}})\right] Y_{l m}^{*}(\hat{\mathbf{n}}) d \Omega \\
& =\sqrt{\frac{(l-|s|) !}{(l+|s|) !} \int\left[\partial^{s}{ }_{-s} A(\hat{\mathbf{n}})\right] Y_{l m}^{*}(\hat{\mathbf{n}}) d \Omega .}
\end{aligned}
$$

This shows that the coefficients $a_{l m}^{A}$ have the desired property of being invariant under a rotation of the coordinate system around $\hat{\mathbf{n}}$. We can then define $E$ and $B$ components through

$$
\begin{gathered}
a_{l m}^{A}=a_{l m}^{A E}+i a_{l m}^{A B}, \quad a_{l m}^{A E}=\frac{1}{2}\left(a_{l m}^{A}+a_{l m}^{A *}\right), \\
a_{l m}^{A B}=\frac{1}{2 i}\left(a_{l m}^{A}-a_{l m}^{A *}\right) .
\end{gathered}
$$

Under a parity transformation $\left(\hat{\mathbf{n}} \rightarrow \hat{\mathbf{n}}^{\prime}=-\hat{\mathbf{n}}\right), Y_{l m} \rightarrow$ $(-1)^{l} Y_{l m}$. Hence, Eq. (A9) yields ${ }_{s} Y_{l m} \rightarrow(-1)^{l}{ }_{s} Y_{l m}=$ $(-1)^{l+s}\left[{ }_{s} Y_{l-m}\right]^{*}$, and $E$ and $B$ components transform under parity as

$$
\begin{gathered}
a_{l m}^{A} \rightarrow(-1)^{l+s} a_{l-m}^{A *}, \quad a_{l m}^{A E} \rightarrow(-1)^{l+s} a_{l-m}^{A E}, \\
a_{l m}^{A B} \rightarrow-(-1)^{l+s} a_{l-m}^{A B} .
\end{gathered}
$$


Thus, the $E$-component coefficients transform as expected of a spin- $s$ quantity derived from a scalar perturbation; for example, a vector given by a gradient $\mathcal{B}_{i}=\partial_{\perp i} f$, whose components ${ }_{ \pm 1} \mathcal{B}$ transform as spin \pm 1 fields, is parity odd (just like the electric field). On the other hand, the $B$ component picks up an additional sign (parity even, just like the magnetic field).

\section{Angular power spectra of spin-s functions}

The general procedure for obtaining the spherical harmonic coefficients and angular power spectrum for a spin- $s$ quantity ${ }_{s} A$ is as follows. The starting point is a relation between ${ }_{s} A(\hat{\mathbf{n}}, \tilde{z})$ and the metric perturbations integrated over the (unperturbed) past light cone:

$$
{ }_{s} A(\hat{\mathbf{n}}, \tilde{z})=\int_{0}^{\tilde{\chi}} d \chi G[\chi, h(\chi \hat{\mathbf{n}}, \eta)]
$$

where $\eta=\eta_{0}-\chi, h(\mathbf{x}, \eta)$ stands for a single polarization state of any given metric perturbation, and the kernel $G$ is a function of $\chi$ and $h$ and its derivatives. Throughout, we suppress the polarization index, although all polarization states of course need to be summed in Eq. (A17). The goal is to derive the contribution of a single Fourier mode of the metric perturbation, and to subsequently add up the contributions of all Fourier modes. For a scalar quantity $(s=0)$, such as a density or temperature, this calculation is straightforward since a scalar is invariant under a general rotation of the coordinate system. Thus, we can always align a given Fourier mode with the $z$ axis before summing up the contributions. For a general spin- $s$ quantity ${ }_{s} A$, this is not possible. However, we can use the spin-raising and -lowering operators defined above to create a scalar quantity $\bar{\partial}_{s}^{s} A$ (for $s>0$ ) which allows us to easily sum up the contributions of different Fourier modes. The results of the previous section then immediately tell us how the resulting spherical harmonic coefficients of $\overline{\mathrm{d}}^{s} A$ are related to those of ${ }_{s} A$.

In detail, the calculation proceeds as follows:

(1) Evaluate the contribution ${ }_{s} A(\hat{\mathbf{n}}, \mathbf{k})$ from a single plane-wave perturbation with a single circular polarization, with wave vector $\mathbf{k}$ aligned with the $z$ axis. ${ }_{s} A(\hat{\mathbf{n}}, \mathbf{k})$ is a function of $\hat{\mathbf{n}}$, usually written in terms of $\mu \equiv \hat{\mathbf{n}} \cdot \hat{\mathbf{z}}$ and azimuthal angle $\phi$ :

$$
{ }_{s} A(\hat{\mathbf{n}}, \mathbf{k})=\int_{0}^{\tilde{\chi}} d \chi \tilde{G}(\chi, k, \mu) h(\mathbf{k}) e^{i r \phi} e^{i x \mu},
$$

where $h(\mathbf{k})$ is the Fourier amplitude of the mode at some reference epoch, $x=k \chi$ and $r$ is an integer. In particular, $r=0$ if $h$ is a scalar metric perturbation, $r= \pm 1$ for a vector perturbation, and $r= \pm 2$ for a tensor perturbation, depending on the polarization state. $\tilde{G}$ is an ordinary function obtained from $G[\chi, h]$ by replacing $\partial_{i}$ with $i k_{i}$, and pulling out $h(\mathbf{k})$. Note that $\tilde{G}$ thus contains the transfer function of the metric perturbation, relating $h$ at the reference epoch to $h$ at conformal time $\eta=\eta_{0}-\chi$

(2) Apply spin-raising or -lowering operators to obtain a scalar quantity, $\bar{\partial}_{s}^{s} A(\hat{\mathbf{n}}, \mathbf{k})$ if $s>0$, $\partial^{|s|}{ }_{s} A(\hat{\mathbf{n}}, \mathbf{k})$ if $s<0$. This quantity is a scalar on the sphere. By virtue of the exponential $e^{i x \mu}$, we can turn derivatives with respect to $\mu$ into powers of $i x$, and powers of $\mu$ into derivatives with respect to $i x$. We can then write

$$
\begin{aligned}
& \bar{\partial}_{s}^{s} A(\hat{\mathbf{n}}, \mathbf{k}) \\
& \quad=\int_{0}^{\tilde{\chi}} d \chi \sum_{i} W_{i}(\chi) \hat{Q}_{i}(\chi)\left(1-\mu^{2}\right)^{|r| / 2} e^{i r \phi} e^{i x \mu} h(\mathbf{k}),
\end{aligned}
$$

where $\hat{Q}_{i}(x)$ are derivative operators in $x, W_{i}(\chi)$ are coefficient functions, and we have pulled out a factor of $\left(1-\mu^{2}\right)^{|r| / 2}$ for later convenience. Note that since the $\hat{Q}_{i}(x)$ are constructed out of powers of $i k=i x / \chi$ and $\partial / \partial(i x)$, the terms involving even powers of $x, \partial_{x}$ are real, while those involving odd powers are imaginary. Hence, $\hat{Q}_{i}^{*}(x)=\hat{Q}_{i}(-x)$.

(3) Since the angular dependence is now entirely in the factor $\left(1-\mu^{2}\right)^{|r| / 2} e^{i r \phi} e^{i x \mu}$, we can straightforwardly expand this scalar quantity in terms of the standard spherical harmonics $Y_{l m}$ following Eq. (A14). The following relation which we prove in Appendix A 2 is useful:

$$
\begin{aligned}
& \int d \Omega Y_{l m}^{*}\left(1-\mu^{2}\right)^{|r| / 2} e^{i r \phi} e^{i x \mu} \\
& =\sqrt{4 \pi(2 l+1)} \sqrt{\frac{(l+|r|) !}{(l-|r|) !}} i^{r} l^{l} \frac{j_{l}(x)}{x^{|r|}} \delta_{m r},
\end{aligned}
$$

where $r$ is an integer. With this, we obtain

$$
\begin{aligned}
a_{l m}^{A}(k)= & \delta_{m r} \sqrt{4 \pi(2 l+1)} \sqrt{\frac{(l-|s|) !(l+|r|) !}{(l+|s|) !}}(-1)^{s} i^{l} \\
& \times \int_{0}^{\tilde{\chi}} d \chi \sum_{i} W_{i}(\chi) i^{r} \hat{Q}_{i}(x) \frac{j_{l}(x)}{x^{|r|}} h(\mathbf{k}) .
\end{aligned}
$$

(4) Following Eq. (A16), we can now separate the $E$ - and $B$-mode contributions:

$$
\begin{aligned}
a_{l m}^{A E}(k)= & \delta_{m r} \sqrt{4 \pi(2 l+1)} \sqrt{\frac{(l-|s|) !(l+|r|) !}{(l+|s|) !}}(-1)^{s} i^{l} \\
& \times \int_{0}^{\tilde{\chi}} d \chi \sum_{i} W_{i}(\chi) i^{r} \operatorname{Re} \hat{Q}_{i}(x) \frac{j_{l}(x)}{x^{|r|}} h(\mathbf{k})
\end{aligned}
$$




$$
\begin{aligned}
a_{l m}^{A B}(k)= & \delta_{m r} \sqrt{4 \pi(2 l+1)} \sqrt{\frac{(l-|s|) !(l+|r|) !}{(l+|s|) !}}(-1)^{s} i^{l} \\
& \times \int_{0}^{\tilde{\chi}} d \chi \sum_{i} W_{i}(\chi) i^{r} \operatorname{Im} \hat{Q}_{i}(x) \frac{j_{l}(x)}{x^{|r|}} h(\mathbf{k}) .
\end{aligned}
$$

These constitute the multipole coefficients of $E$ and $B$ modes of ${ }_{s} A$.

The angular power spectra are straightforwardly obtained by taking the expectation value of quadratic combinations of $a_{l m}^{A X}(k)$, where $X=E$, $B$, summing over $m$, and integrating over $(2 \pi)^{-6} d^{3} k d^{3} k^{\prime}$. Note that since we derived the multipole coefficients through the scalar quantity $\bar{\partial}^{s} A(\hat{\mathbf{n}}, \mathbf{k})$ which is invariant under rotations of the coordinate system, we can always align the Fourier mode with the $z$ axis, so that Eqs. (A21)-(A23) remain valid. Thus,

$$
\begin{gathered}
C_{A}^{X X}(l) \\
=\frac{1}{2 l+1} \sum_{m=-l}^{l} \int \frac{d^{3} k}{(2 \pi)^{3}} \int \frac{d^{3} k^{\prime}}{(2 \pi)^{3}}\left\langle a_{l m}^{A X}(k) a_{l m}^{A X *}\left(k^{\prime}\right)\right\rangle \\
=\frac{2}{\pi} \frac{(l-|s|) !(l+|r|) !}{(l+|s|) !(l-|r|) !} N_{P} \int k^{2} d k P_{h}(k)\left|F_{l}^{X}(k)\right|^{2} \\
F_{l}^{E}(k)=\int_{0}^{\tilde{\chi}} d \chi \sum_{i} W_{i}(\chi) \operatorname{Re} \hat{Q}(k \chi) \frac{j_{l}(k \chi)}{(k \chi)^{|r|}} \\
F_{l}^{B}(k)=\int_{0}^{\tilde{\chi}} d \chi \sum_{i} W_{i}(\chi) \operatorname{Im} \hat{Q}(k \chi) \frac{j_{l}(k \chi)}{(k \chi)^{|r|}} .
\end{gathered}
$$

Here, $P_{h}(k)$ is the power spectrum of $h(\mathbf{k})$ at the chosen reference epoch, $N_{P}$ denotes the number of polarization states, and we have assumed that the different polarization states have independent phases and equal power spectra.

Equation (A24) is a general expression for the $E / B$-mode angular power spectra of a spin-s observable induced by a spin- $r$ metric perturbation, which is straightforward to evaluate once an expression of the form Eq. (A19) is given. Note that for $r=s=0$ and $N_{P}=1$, we recover the usual result for scalar observables induced by scalar perturbations. In the following, and in the related papers [21,24], we will apply this result for $s=0, \pm 1$ and \pm 2 , as well as $r=0, \pm 2$.

\section{Proof of Eq. (A20)}

The useful relation Eq. (A20) is easily proven by induction over $r$. First, the case $r=0$,

$$
\int d \Omega Y_{l m}^{*} e^{i x \mu}=\sqrt{4 \pi(2 l+1)} i^{l} j_{l}(x) \delta_{m 0},
$$

follows immediately from our definition of the spherical harmonics, Eq. (A1), and the partial wave expansion

$$
e^{i x \cos \theta}=\sum_{l}(2 l+1) i^{l} j_{l}(x) P_{l}(\cos \theta) .
$$

Further, the definition of spherical harmonics, Eq. (A1), yields

$$
\begin{gathered}
\int d \Omega Y_{l m}^{*}\left(1-\mu^{2}\right)^{|r| / 2} e^{i r \phi} e^{i x \mu} \\
=\epsilon_{r} \sqrt{4 \pi(2 l+1)} \sqrt{\frac{(l-|r|) !}{(l+|r|) !}} \delta_{r m} I_{l}^{|r|}(x), \\
I_{l}^{|r|}(x)=\frac{1}{2} \int_{-1}^{1} d \mu\left(1-\mu^{2}\right)^{|r| / 2} P_{l}^{|r|}(\mu) e^{i x \mu},
\end{gathered}
$$

where

$$
P_{l}^{m}(x)=(-1)^{m}\left(1-x^{2}\right)^{m / 2} \frac{d^{m}}{d x^{m}} P_{l}(x) \quad(m \geq 0)
$$

is the associated Legendre polynomial. Comparing with Eq. (A20), the conjecture to prove is thus

$$
I_{l}^{|r|}(x)=\frac{(l+|r|) !}{(l-|r|) !} i^{|r|+l} \frac{j_{l}(x)}{x^{|r|}} .
$$

We now proceed to the proof by induction, assuming that Eq. (A30) holds for some $r>0$ (without loss of generality). Using one partial integration on $I_{l}^{r+1}(x)$ we obtain

$$
\begin{aligned}
I_{l}^{r+1}(x) & =\frac{1}{2} \int_{-1}^{1} d \mu\left(1-\mu^{2}\right)^{(r+1) / 2}(-1)^{(r+1)}\left(1-\mu^{2}\right)^{(r+1) / 2}\left[\frac{d^{r+1}}{d \mu^{r+1}} P_{l}(\mu)\right] e^{i x \mu} \\
& =\frac{1}{2} \int_{-1}^{1} d \mu(-1)^{r}\left[\frac{d^{r}}{d \mu^{r}} P_{l}(\mu)\right] \frac{d}{d \mu}\left[\left(1-\mu^{2}\right)^{r+1} e^{i x \mu}\right] \\
& =\frac{1}{2} \int_{-1}^{1} d \mu(-1)^{r}\left(1-\mu^{2}\right)^{r}\left[\frac{d^{r}}{d \mu^{r}} P_{l}(\mu)\right]\left[-2 \mu(r+1)+\left(1-\mu^{2}\right) i x\right] e^{i x \mu}=\left[2(r+1) \partial_{x}+x\left(1+\partial_{x}^{2}\right)\right] i I_{l}^{r}(x),
\end{aligned}
$$

where in the third line we have converted powers of $i \mu$ to derivatives $\partial_{x}$. Now we use that, by assumption, Eq. (A30) holds for $r$, which leads to 


$$
I_{l}^{r+1}(x)=\frac{(l+r) !}{(l-r) !} i^{r+1+l}\left[2(r+1) \partial_{x}+x\left(1+\partial_{x}^{2}\right)\right] \frac{j_{l}(x)}{x^{r}} .
$$

Straightforward algebra, together with the differential equation satisfied by spherical Bessel functions, $j_{l}^{\prime \prime}=$ $-2 j_{l}^{\prime} / x+\left[l(l+1) / x^{2}-1\right] j_{l}$ leads to

$$
I_{l}^{r+1}(x)=2 \frac{(l+r+1) !}{(l-r-1) !} i^{r+l+1} \frac{j_{l}(x)}{x^{r+1}},
$$

which proves the conjecture Eq. (A30).

\section{APPENDIX B: GEODESIC EQUATION AND DISPLACEMENTS}

In this appendix, we outline the derivation of the displacements Eqs. (34)-(37) in the general gauge given by Eq. (2) and (19). This is a generalization of the derivation in Ref. [11] (which considered the sc gauge), and a special case of the treatment in Ref. [37]. Choosing the (zerothorder) comoving distance as the affine parameter, the photon momentum can be written as

$$
\frac{d x^{\mu}}{d \chi}=\left(-1+\delta \nu, \hat{n}^{i}+\delta e^{i}\right) .
$$

The geodesic equation then becomes

$$
\frac{d}{d \chi} \frac{d x^{\mu}}{d \chi}+\Gamma_{\alpha \beta}^{\mu} \frac{d x^{\alpha}}{d \chi} \frac{d x^{\beta}}{d \chi}=0
$$

The zeroth-order parts just yield $d x^{\mu} / d \chi=$ const. We now turn to the first-order part. The temporal component gives

$$
\begin{aligned}
& \frac{d}{d \chi} \delta \nu+\Gamma_{\alpha \beta}^{0} \frac{d x^{\alpha}}{d \chi} \frac{d x^{\beta}}{d \chi}=0 \\
& \frac{d}{d \chi} \delta \nu+A^{\prime}-2 A_{, i} \hat{n}^{i}+\left(\frac{1}{2} h_{i j}^{\prime}+B_{(i, j)}\right) \hat{n}^{i} \hat{n}^{j}=0 \\
& \frac{d}{d \chi}(\delta \nu-2 A)=A^{\prime}-\frac{1}{2} h_{\|}^{\prime}-\partial_{\|} B_{\|} .
\end{aligned}
$$

The spatial components yield

$$
\begin{gathered}
\frac{d}{d \chi} \delta e^{i}+\Gamma_{\alpha \beta}^{i} \frac{d x^{\alpha}}{d \chi} \frac{d x^{\beta}}{d \chi}=0 \\
\frac{d}{d \chi} \delta e^{i}+A^{, i}-B^{i \prime}-h^{\prime i}{ }_{j} \hat{n}^{j}-\left(B_{j, i}-B_{i, j}\right) \hat{n}^{j}+\frac{1}{2}\left(h_{j, k}^{i}+h_{k, j}^{i}-h_{j k}{ }^{i}\right) \hat{n}^{j} \hat{n}^{k}=0 \\
\frac{d}{d \chi}\left(\delta e^{i}+B^{i}+h^{i}{ }_{j} \hat{n}^{j}\right)=-A^{, i}+\partial_{i} B_{\|}-B_{\perp i}+\frac{1}{2} h_{\|}{ }^{i}-\frac{1}{\chi} \mathcal{P}^{i j} h_{j k} \hat{n}^{k}
\end{gathered}
$$

Next, we need to obtain the initial conditions at the observer for the quantities $\delta \nu, \delta e^{i}$. For this, we consider an orthonormal tetrad $\left(e^{a}\right)_{\mu}$, defined through

$$
g^{\mu \nu}\left(e^{a}\right)_{\mu}\left(e^{b}\right)_{\nu}=\eta^{a b} .
$$

To zeroth order, we set $\left(e^{b}\right)_{\mu}=a^{2} \eta_{\mu}^{b}$. Using Eq. (2), this yields at first order

$$
\begin{aligned}
-1 & =g^{\mu \nu}\left(e^{0}\right)_{\mu}\left(e^{0}\right)_{\nu}=a^{-2}(-1+2 A)\left[\left(e^{0}\right)_{0}\right]^{2} \\
\delta^{i j} & =g^{\mu \nu}\left(e^{i}\right)_{\mu}\left(e^{j}\right)_{\nu}=a^{-2}\left(e^{i}\right)_{k}\left(e^{j}\right)_{l}\left(\delta^{k l}-h^{k l}\right) \\
0 & =g^{\mu \nu}\left(e^{0}\right)_{\mu}\left(e^{i}\right)_{\nu} \\
& =a^{-2}\left[-\left(e^{0}\right)_{0}\left(e^{i}\right)_{0}-B^{j}\left(e^{i}\right)_{j}\left(e^{0}\right)_{0}+\left(e^{0}\right)_{j}\left(e^{i}\right)^{j}\right] .
\end{aligned}
$$

The first line yields $\left(e^{0}\right)_{0}=a(-1-A)$, while the second line yields $\left(e^{k}\right)_{l}=a\left(\delta_{l}^{k}+h_{l}^{k} / 2\right)$. With this, the third line becomes

$$
0=a\left(e^{i}\right)_{0}+a^{2} B^{i}+a\left(e^{0}\right)_{i} .
$$

Further, we require that the spatial hypersurfaces defined by $\left(e^{i}\right)_{\mu}$ be orthogonal to the 4-velocity of comoving observers [Eq. (19)]:

$$
0=\left(e^{i}\right)_{\mu} u^{\mu}=\left(e^{i}\right)_{0} a^{-1}(1-A)+v^{i} .
$$

Hence, we have $\left(e^{i}\right)_{0}=-a v^{i}$, and Eq. (B7) yields $\left(e^{0}\right)_{i}=$ $a\left(v_{i}-B_{i}\right)$. We thus have

$$
\begin{aligned}
& \left(e^{0}\right)_{\mu}=a\left(-1-A, v_{i}-B_{i}\right) \\
& \left(e^{j}\right)_{\mu}=a\left(-v^{j}, \delta^{j}{ }_{i}+\frac{1}{2} h^{j}{ }_{i}\right) .
\end{aligned}
$$

Comparing the first line with Eq. (19), we indeed see that $\left(e^{0}\right)_{\mu}=u_{\mu}$, as desired.

We now require that the normalized photon momentum at the observer, $\hat{p}^{\mu}=\left(-1+\delta \nu_{o}, \hat{n}^{i}+\delta e_{o}^{i}\right)$, measured with respect to this tetrad has the components $\left(1, \hat{n}^{i}\right)$ (note that for our choice of affine parameter, the photon momentum is past directed). Setting $a_{o}=1$ without loss of generality, we obtain

$$
\begin{aligned}
& 1=\left(e^{0}\right)_{\mu} p^{\mu}=1+A_{o}-\delta \nu_{o}+\left(v_{i o}-B_{i o}\right) \hat{n}^{i} \\
& \hat{n}^{i}=\left(e^{i}\right)_{\mu} p^{\mu}=\hat{n}^{i}+v_{o}^{i}+\delta e_{o}^{i}+\frac{1}{2}\left(h_{j}^{i}\right)_{o} \hat{n}^{j} .
\end{aligned}
$$

Note that the first condition implies that a locally emitted photon is observed with unperturbed redshift [see Eq. (B16)]. Hence,

$$
\delta \nu_{0}=A_{o}+v_{\| o}-B_{\| o}, \quad \delta e_{o}^{i}=-v_{o}^{i}-\frac{1}{2}\left(h_{j}^{i}\right)_{o} \hat{n}^{j} .
$$


Note the aberration term $-v_{o}^{i}$ in $\delta e_{o}^{i}$. We can now integrate the geodesic equations given these initial conditions:

$$
\begin{aligned}
\delta \nu(\chi)= & 2 A(\chi)+\int_{0}^{\chi} d \chi^{\prime}\left[A^{\prime}-\frac{1}{2} h_{\|}^{\prime}-\partial_{\|} B_{\|}\right]+\text {const } \\
= & -A_{o}+v_{\| o}-B_{\| o}+2 A(\chi) \\
& +\int_{0}^{\chi} d \chi^{\prime}\left[A^{\prime}-\frac{1}{2} h_{\|}^{\prime}-\partial_{\|} B_{\|}\right] .
\end{aligned}
$$

The spatial component yields

$$
\begin{aligned}
\delta e^{i}(\chi)= & \frac{1}{2}\left(h^{i}{ }_{j}\right)_{o} \hat{n}^{j}-v_{o}^{i}+B_{o}^{i}-B^{i}(\chi)-h^{i}{ }_{j}(\chi) \hat{n}^{j} \\
& +\int_{0}^{\chi} d \chi^{\prime}\left[-A^{, i}+\hat{n}^{j} B_{j, i}+\frac{1}{2} h_{j k}{ }^{,} \hat{n}^{j} \hat{n}^{k}\right] .
\end{aligned}
$$

Integrating again yields the temporal and spatial displacements:

$$
\begin{aligned}
\delta x^{0}(\tilde{\chi})= & \int_{0}^{\tilde{\chi}} d \chi d \nu(\chi) \\
= & {\left[-A_{o}+v_{\| o}-B_{\| o}\right] \tilde{\chi}+} \\
& \int_{0}^{\tilde{\chi}} d \chi\left[2 A(\chi)+(\tilde{\chi}-\chi)\left\{A^{\prime}-\frac{1}{2} h_{\|}^{\prime}-\partial_{\|} B_{\|}\right\}\right] \\
\delta x^{i}(\tilde{\chi})= & \int_{0}^{\tilde{\chi}} d \chi \delta e^{i}(\chi) \\
= & {\left[\frac{1}{2}\left(h^{i}{ }_{j}\right)_{o} \hat{n}^{j}+B_{o}^{i}-v_{o}^{i}\right] \tilde{\chi} } \\
& +\int_{0}^{\tilde{\chi}} d \chi\left[-B^{i}-h^{i}{ }_{j} \hat{n}^{j}+(\tilde{\chi}-\chi)\right. \\
& \left.\times\left\{-A^{, i}+\hat{n}^{j} B_{j, i}+\frac{1}{2} h_{j k}{ }^{i} \hat{n}^{j} \hat{n}^{k}\right\}\right] .
\end{aligned}
$$

Next, we need to evaluate the scale factor and affine parameter at emission, by requiring that the observed photon frequency match the redshift $\tilde{z}$. Since the photon momentum is given by Eq. (B1), we have

$$
\begin{aligned}
1+\tilde{z} & =\frac{\left(a^{-2} u_{\mu} d x^{\mu} / d \chi\right)_{e}}{\left(a^{-2} u_{\mu} d x^{\mu} / d \chi\right)_{o}} \\
& =\frac{a^{-1}\left(x^{0}\right)\left(1+A-\delta \nu+v_{\|}-B_{\|}\right)_{e}}{\left(1+A-\delta \nu+v_{\|}-B_{\|}\right)_{o}},
\end{aligned}
$$

where a subscript $e$ denotes the emission point, and we have decomposed $B_{i}=B_{\|} \hat{n}_{i}+B_{\perp i}$. The initial conditions for $\delta \nu$ imply that the denominator is 1 , and we obtain

$$
\frac{a\left(x^{0}\right)}{\tilde{a}}=1+A-\delta \nu+v_{\|}-B_{\|} \equiv 1+\Delta \ln a,
$$

where all quantities on the right-hand side are evaluated at emission, and we have defined the perturbation to the $\operatorname{logarithm}$ of the scale factor at emission, $\Delta \ln a$. Explicitly,

$$
\begin{aligned}
\Delta \ln a= & A-\delta \nu+v_{\|}-B_{\|} \\
= & A_{o}-A+v_{\|}-v_{\| o} \\
& +\int_{0}^{\tilde{\chi}} d \chi\left[-A^{\prime}+\frac{1}{2} h_{\|}^{\prime}+B_{\|}^{\prime}\right] .
\end{aligned}
$$

Since $\tilde{a}=1 /(1+\tilde{z})$, and $a\left(x^{0}\right)=1 /(1+\bar{z})$ where $\bar{z}$ is the redshift one would observe for the same source in an unperturbed Universe, we can write this as

$$
1+\tilde{z}=(1+\bar{z})(1+\Delta \ln a) .
$$

The perturbation to the conformal time at emission has two contributions, from the temporal displacement $\delta x^{0}$ and from the perturbation to the affine parameter from its zeroth-order value, $\tilde{\chi}$ :

$$
a\left(x^{0}\right)=\tilde{a}+\tilde{a} \frac{\partial \ln a}{\partial \eta}\left[\delta x^{0}-\delta \chi\right] .
$$

Equation (B19) thus yields

$$
1=1-\tilde{a} H(\tilde{z})\left[\delta x^{0}-\delta \chi\right]+\Delta \ln a,
$$

so that

$$
\delta \chi=\delta x^{0}-\frac{1+\tilde{z}}{H(\tilde{z})} \Delta \ln a .
$$

We can now assemble the total line-of-sight deflection:

$$
\begin{aligned}
\Delta x_{\|} & =\delta x^{i} \hat{n}_{i}+\delta \chi=\delta x_{\|}+\delta x^{0}-\frac{1+\tilde{z}}{H(\tilde{z})} \Delta \ln a \\
& =\int_{0}^{\tilde{\chi}} d \chi\left[A-B_{\|}-\frac{1}{2} h_{\|}\right]-\frac{1+\tilde{z}}{H(\tilde{z})} \Delta \ln a .
\end{aligned}
$$

For the transverse deflection, we obtain

$$
\begin{aligned}
\Delta x_{\perp}^{i}= & \delta x^{i}-\hat{n}^{i} \delta x_{\|} \\
= & {\left[\frac{1}{2} \mathcal{P}^{i j}\left(h_{j k}\right)_{o} \hat{n}^{k}+B_{\perp o}^{i}-v_{\perp o}^{i}\right] \tilde{\chi} } \\
& +\int_{0}^{\tilde{\chi}} d \chi\left[-B_{\perp}^{i}-\mathcal{P}^{i j} h_{j k} \hat{n}^{k}+(\tilde{\chi}-\chi)\right. \\
& \left.\times\left\{-\partial_{\perp}^{i} A+\hat{n}^{k} \partial_{\perp}^{i} B_{k}+\frac{1}{2}\left(\partial_{\perp}^{i} h_{j k}\right) \hat{n}^{j} \hat{n}^{k}\right\}\right],
\end{aligned}
$$

which can be further manipulated to yield Eq. (36).

\section{APPENDIX C: TEST CASES FOR THE SHEAR}

In this appendix, we consider test cases in order to validate the expression for the shear, Eq. (65) in Sec. VII B. For a larger set of test cases applied to scalar quantities such as the observed galaxy density and the magnification, see Appendix C in Ref. [11].

The first case is a metric perturbation corresponding to a pure gauge mode, i.e.,

$$
h_{i j}(\mathbf{x}, \eta)=A_{i j}+B_{i j k} x^{k},
$$

where $A_{i j}$ and $B_{i j k}$ are constant and symmetric in $i$ and $j$. Such a metric perturbation can be obtained through a coordinate 
transform $x^{i} \rightarrow \hat{x}^{i}=x^{i}-A_{i j} x^{j} / 2-B_{i j k} x^{j} x^{k} / 4$. Choosing the observer to be at the origin, we have

$$
\begin{gathered}
h_{ \pm o}=A_{ \pm}, \quad h_{ \pm s}=A_{ \pm}+B_{ \pm k} \tilde{x}^{k}, \\
\int_{0}^{\tilde{\chi}} \frac{d \chi}{\tilde{\chi}} h_{ \pm}=A_{ \pm}+\frac{1}{2} B_{ \pm k} \tilde{x}^{k},
\end{gathered}
$$

where $B_{ \pm k}=m_{\mp}^{i} m_{\mp}^{j} B_{i j k}$. Since $\partial_{i} \partial_{j} h_{k l}=0$ and $\partial_{i} h_{k l}=$ $B_{k l i}=$ const, Eq. (65) then yields

$$
{ }_{ \pm 2} \gamma(\hat{\mathbf{n}})=-\frac{1}{2} A_{ \pm}-\frac{1}{2} A_{ \pm}-\frac{1}{2} B_{ \pm k} \tilde{x}^{k}+A_{ \pm}+\frac{1}{2} B_{ \pm k} \tilde{x}^{k}=0 .
$$

Thus, a gauge mode does not contribute to the shear.

Further possible test cases are a perturbed expansion history and spatial curvature. Both cases correspond to isotropic Universes, and the observed shear should thus be 0 . The former case is described by a metric perturbation of the form $h_{i j}=A(\eta) \delta_{i j}$. Since this implies that $\partial_{i} h_{j k}=0$ and $h_{ \pm}=0$, Eq. (65) implies ${ }_{ \pm 2} \gamma=0$. Spatial curvature is described by a metric perturbation $h_{i j}=-K / 4 x_{k} x^{k} \delta_{i j}$. In this case, $h_{ \pm}=0, \partial_{i} h_{k l}=-K / 2 x_{i} \delta_{k l}$, and $\partial_{i} \partial_{j} h_{k l}=$ $-K / 2 \delta_{i j} \delta_{k l}$. Thus,

$$
\begin{aligned}
\left(m_{+}^{i} m_{\mp}^{j} \partial_{i} \partial_{j} h_{k l}\right) \hat{n}^{k} \hat{n}^{l} & =-\frac{K}{2}\left(m_{+}^{i} m_{\mp i}\right)^{2}=0 \\
\hat{n}^{l} m_{+}^{k} m_{+}^{i} \partial_{i} h_{k l} & =-\frac{K}{2}\left(m_{+}^{i} x_{i}\right) \hat{n}_{k} m_{+}^{k}=0 .
\end{aligned}
$$

This implies that ${ }_{ \pm 2} \gamma=0$ for spatial curvature as well. There is however one test case where the shear is nontrivial, which we will consider next.

\section{Bianchi I cosmology}

A Bianchi I cosmology is an anisotropically expanding Universe. Following Ref. [11], we choose the 3 -axis to be unperturbed, while the scale factors along the 1- and 2-axes are perturbed in the following way:

$$
\begin{aligned}
& a_{1}(\eta)=a(\eta)\left[1+s_{1}(\eta)-s_{3}(\eta)\right] \\
& a_{2}(\eta)=a(\eta)\left[1+s_{2}(\eta)-s_{3}(\eta)\right] \\
& a_{3}(\eta)=a(\eta),
\end{aligned}
$$

where $s_{1}(\eta)+s_{2}(\eta)+s_{3}(\eta)=0, \quad$ and $\quad s_{i}\left(\eta_{0}\right)=0$. Relaxing either of these conditions leads to cases we have studied above (perturbed expansion history and pure gauge mode). The nonzero components of $h_{i j}$ are then given by

$$
h_{11}=2\left[s_{1}(\eta)-s_{3}(\eta)\right], \quad h_{22}=2\left[s_{2}(\eta)-s_{3}(\eta)\right] .
$$

Let us consider two lines of sight close to the unperturbed 3axis. Specifically, we consider photon 4-momenta at the observer given by $p_{\mu}^{(1)}=(-1,-\varsigma, 0,-1)$ and $p_{\mu}^{(2)}=$ $(-1,0,-\varsigma, 0,-1)$, where $s$ is the infinitesimal angle with the 3-axis. By following back these geodesics, one can straightforwardly derive the angular diameter distances along the 3-axis, for an object extended along the 1- and 2-axes [11]:

$$
\begin{aligned}
D_{A, \text { phys }, a}(\eta)= & a(\eta)\left(\eta_{0}-\eta\right)\left[1+s_{a}(\eta)-s_{3}(\eta)\right. \\
& \left.+\frac{2}{\eta_{0}-\eta} \int_{\eta}^{\eta_{0}} d \eta^{\prime}\left(s_{3}\left(\eta^{\prime}\right)-s_{a}\left(\eta^{\prime}\right)\right)\right],
\end{aligned}
$$

where $a=1,2$, and $\eta$ is the conformal time of emission. The observed ellipticity of galaxies, designed as an estimator for shear, can be written as

$$
\begin{aligned}
\epsilon_{1} & =\frac{1}{2} \frac{I_{11}-I_{22}}{I_{11}+I_{22}}=\frac{1}{2} \frac{D_{A, \text { phys }, 1}^{-2}-D_{A, \text { phys }, 2}^{-2}}{D_{A, \text { phys }, 1}^{-2}+D_{A, \text { phys }, 2}^{-2}} \\
\epsilon_{2} & =\frac{I_{12}}{I_{11}+I_{22}}=0,
\end{aligned}
$$

where $I_{i j}$ are the quadrupole moments of the galaxy's light distribution, which scale as $D_{A \text {, phys }}^{-2}$. Other definitions are possible; however all of them agree at linear order. $\epsilon_{2}$ vanishes, since a ray with $p_{\mu}=(-1,-\varsigma / \sqrt{2}, \varsigma / \sqrt{2},-1)$, propagating at $+45^{\circ}$ azimuthal angle to the 1-axis, yields the same angular diameter distance as a ray with $p_{\mu}=$ $(-1,-\varsigma / \sqrt{2},-\varsigma / \sqrt{2},-1)$, propagating at $-45^{\circ}$ angle. Assuming that the galaxies are on average round $\left(\left\langle\epsilon_{i}\right\rangle=\right.$ $0)$, i.e., that they are not directly influenced by the anisotropic expansion, we obtain

$$
\begin{aligned}
\epsilon_{1}= & \frac{1}{4}\left[-2 s_{1}+2 s_{3}+2 s_{2}-2 s_{3}\right. \\
& \left.-\frac{4}{\eta_{0}-\eta} \int_{\eta}^{\eta_{0}} d \eta^{\prime}\left[-s_{1}\left(\eta^{\prime}\right)+s_{2}\left(\eta^{\prime}\right)\right]\right] \\
= & \frac{1}{2}\left[s_{2}(\eta)-s_{1}(\eta)\right]+\frac{1}{\eta_{0}-\eta} \int_{\eta}^{\eta_{0}} d \eta^{\prime}\left[s_{1}\left(\eta^{\prime}\right)-s_{2}\left(\eta^{\prime}\right)\right] .
\end{aligned}
$$

The extra factor of $1 / 2$ is due to the sum of moments in the denominator in the definition of $\epsilon_{1}$. In order to compare with Eq. (65), we use

$$
h_{ \pm}=\frac{1}{2}\left(h_{11}-h_{22} \pm 2 i h_{12}\right)=s_{1}(\eta)-s_{2}(\eta) .
$$

We thus have $h_{ \pm o}=0$, and Eq. (65) yields for the shear along the 3 -axis

$$
\begin{aligned}
\gamma_{1} \pm i \gamma_{2}= & -\frac{1}{2}\left(s_{1}(\eta)-s_{2}(\eta)\right) \\
& +\int_{0}^{\tilde{\chi}} \frac{d \chi}{\tilde{\chi}}\left[s_{1}(\eta(\chi))-s_{2}(\eta(\chi))\right] \\
= & \frac{1}{2}\left(s_{2}(\eta)-s_{1}(\eta)\right) \\
& +\frac{1}{\eta_{0}-\eta} \int_{\eta}^{\eta_{0}} d \eta^{\prime}\left[s_{1}\left(\eta^{\prime}\right)-s_{2}\left(\eta^{\prime}\right)\right],
\end{aligned}
$$

where we have used that ${ }_{ \pm 2} \gamma=\gamma_{1} \pm i \gamma_{2}$ for the coordinates chosen here [see Eq. (50)]. Since this expression is 
real, $\gamma_{2}=0$, and the result is equal to $\gamma_{1}$, which moreover agrees with the correct physical result for $\epsilon_{1}$, Eq. (C9).

\section{Shear from scalar perturbations}

In the derivation leading to Eq. (65), we have not made any assumptions about metric perturbations except that $\delta g_{00}=0=\delta g_{0 i}$ (sc gauge). As a cross-check, we now consider the case of scalar perturbations, where we can write

$$
h_{i j}=2 D \delta_{i j}+2\left(\partial_{i} \partial_{j}-\frac{1}{3} \delta_{i j} \nabla^{2}\right) E
$$

in terms of the 3-scalar perturbations $D$ and $E$ (see Ref. [11]). We thus have $h_{ \pm}=2 m_{\mp}^{i} m_{\mp}^{j} E_{, i j}$ and obtain

$$
\begin{aligned}
{ }_{ \pm 2} \gamma(\hat{\mathbf{n}})= & -m_{+}^{i} m_{+}^{j}\left(E_{, i j}(o)+E_{, i j}(s)\right) \\
& -\int_{0}^{\tilde{\chi}} d \chi\left\{(\tilde{\chi}-\chi) \frac{\chi}{\tilde{\chi}} m_{+}^{i} m_{\mp}^{j}\right. \\
& \times\left(D_{, i j}-\frac{1}{3} \nabla^{2} E_{, i j}+\partial_{\|}^{2} E_{, i j}\right) \\
& \left.+2\left(1-2 \frac{\chi}{\tilde{\chi}}\right) m_{+}^{i} m_{+}^{j} \partial_{\|} E_{, i j}-\frac{2}{\tilde{\chi}} m_{+}^{i} m_{\mp}^{j} E_{, i j}\right\} .
\end{aligned}
$$

We now consider the contribution of a single plane wave along the $z$ axis,

$$
D(\mathbf{x}, \eta)=D(\mathbf{k}, \eta) e^{i \mathbf{k} \cdot \hat{\mathbf{n}} \chi}=D(\mathbf{k}, \eta) e^{i x \mu},
$$

and similarly for $E$. We can then replace

$$
m_{+}^{i} m_{\mp}^{j} \partial_{i} \partial_{j} \rightarrow-\frac{1}{2}\left(1-\mu^{2}\right) k^{2} .
$$

This yields

$$
\begin{aligned}
{ }_{ \pm 2} \gamma(\mathbf{k}, \hat{\mathbf{n}})= & +\frac{1}{2}\left(1-\mu^{2}\right) k^{2}\left(\left.E\left(\mathbf{k}, \eta_{0}\right) e^{i x \mu}\right|_{x=0}+E(\mathbf{k}, \tilde{\eta}) e^{i \tilde{x} \mu}\right) \\
& +\int_{0}^{\tilde{\chi}} d \chi\left\{(\tilde{\chi}-\chi) \frac{\chi}{\tilde{\chi}} \frac{1}{2}\left(1-\mu^{2}\right) k^{2}(D(\mathbf{k}, \eta)\right. \\
& \left.+\frac{1}{3} k^{2} E(\mathbf{k}, \eta)-\mu^{2} k^{2} E(\mathbf{k}, \eta)\right) \\
& +\left(1-2 \frac{\chi}{\tilde{\chi}}\right) i\left(1-\mu^{2}\right) \mu k^{3} E(\mathbf{k}, \eta) \\
& \left.-k^{2} \frac{1}{\tilde{\chi}}\left(1-\mu^{2}\right) E(\mathbf{k}, \eta)\right\} e^{i x \mu},
\end{aligned}
$$

where $\tilde{\eta}=\eta_{0}-\tilde{\chi}$, and $\tilde{x}=k \tilde{\chi}$. Note that a scalar perturbation produces equal amplitudes of ${ }_{{ }_{2}} \gamma$ : there is no preferred handedness for scalar modes. Correspondingly, since this expression is $\propto e^{i m \phi}$ with $m=0$, the spinlowering and spin-raising actions [Eq. (A8)] become equivalent. We have

$$
\begin{aligned}
\bar{\partial}_{2}^{2} \gamma= & \partial_{-2}^{2} \gamma=\frac{\partial^{2}}{\partial \mu^{2}}\left[\left(1-\mu^{2}\right)_{2} \gamma(\hat{\mathbf{n}}, \mathbf{k})\right] \\
= & \frac{1}{2} k^{2}\left(E\left(\mathbf{k}, \eta_{0}\right)\left[Q_{1}^{S}(x) x^{2} e^{i x \mu}\right]_{x=0}+E(\mathbf{k}, \eta)\left[Q_{1}^{S}(x) x^{2} e^{i x \mu}\right]_{x=k \tilde{\chi}}\right)+\int_{0}^{\tilde{\chi}} d \chi\left\{( \tilde { \chi } - \chi ) \frac { \chi } { \tilde { \chi } } \frac { 1 } { 2 } k ^ { 2 } \left(Q_{1}^{S}(x) D(\mathbf{k}, \eta)\right.\right. \\
& \left.\left.+\frac{1}{3} Q_{1}^{S}(x) k^{2} E(\mathbf{k}, \eta)-Q_{2}^{S}(x) k^{2} E(\mathbf{k}, \eta)\right)-\left(1-2 \frac{\chi}{\tilde{\chi}}\right) \frac{1}{\chi} Q_{3}^{S}(x) k^{2} E(\mathbf{k}, \eta)-\frac{1}{\tilde{\chi}} Q_{1}^{S}(x) k^{2} E(\mathbf{k}, \eta)\right\}\left.x^{2} e^{i x \mu}\right|_{x=k \chi} .
\end{aligned}
$$

Using that

$$
\begin{aligned}
\frac{\partial^{2}}{\partial \mu^{2}}\left[\left(1-\mu^{2}\right)^{2} e^{i x \mu}\right] & =\frac{\partial^{2}}{\partial \mu^{2}}\left[\left(1+\partial_{x}^{2}\right)^{2} e^{i x \mu}\right] \\
& =-\left(1+\partial_{x}^{2}\right)^{2}\left[x^{2} e^{i x \mu}\right], \\
\frac{\partial^{2}}{\partial \mu^{2}}\left[\left(1-\mu^{2}\right)^{2} \mu e^{i x \mu}\right] & =i \partial_{x}\left(1+\partial_{x}^{2}\right)^{2}\left[x^{2} e^{i x \mu}\right], \\
\frac{\partial^{2}}{\partial \mu^{2}}\left[\left(1-\mu^{2}\right)^{2} \mu^{2} e^{i x \mu}\right] & =\partial_{x}^{2}\left(1+\partial_{x}^{2}\right)^{2}\left[x^{2} e^{i x \mu}\right],
\end{aligned}
$$

we obtain the operators $Q_{i}^{S}(x)$ for the scalar case:

$$
\begin{gathered}
Q_{1}^{S}(x)=-\left(1+\partial_{x}^{2}\right)^{2}, \quad Q_{2}^{S}(x)=\left(1+\partial_{x}^{2}\right)^{2} \partial_{x}^{2}, \\
Q_{3}^{S}(x)=x\left(1+\partial_{x}^{2}\right) \partial_{x} .
\end{gathered}
$$

Note that all these operators are real. Hence, following the general derivation in Appendix A 1, there are no parity-odd terms in the scalar contributions to the shear, and thus no $B$ modes as expected.

\section{APPENDIX D: VECTOR}

In this section, we follow the procedure described in Appendix A 1 in order to derive the multipole moments of the vector component ${ }_{ \pm 1} \mathcal{B}$ induced by scalar and tensor perturbations. For scalar perturbations, we work in the $\mathrm{cN}$ gauge; there is no gauge ambiguity for tensor modes. Our goal is to show that scalar perturbations do not contribute to $a_{l m}^{\mathcal{B B}}$, while tensor perturbations do contribute. 


\section{Scalar modes}

We begin with Eq. (46):

$$
\begin{aligned}
\left({ }_{ \pm 1} \mathcal{B}\right)_{\mathrm{cN}}= & -v_{ \pm}+\frac{1+\tilde{z}}{H(\tilde{z})}\left(-\partial_{ \pm} \Psi+\partial_{ \pm}\left[v_{\|}-v_{\| o}\right]\right. \\
& \left.+\int_{0}^{\tilde{\chi}} d \chi \frac{\chi}{\tilde{\chi}} \partial_{ \pm}\left(\Phi^{\prime}-\Psi^{\prime}\right)\right)
\end{aligned}
$$

$$
\begin{aligned}
\left({ }_{ \pm 1} \mathcal{B}\right)_{\mathrm{cN}}(\hat{\mathbf{n}}, \mathbf{k})= & -i k_{ \pm} V(\mathbf{k}, \tilde{\eta}) e^{i \tilde{x} \mu}+\frac{1+\tilde{z}}{H(\tilde{z})}\left(-i k_{ \pm} \Psi(\mathbf{k}, \tilde{\eta}) e^{i \tilde{x} \mu}\right. \\
& \left.+\left(\frac{i k_{ \pm}}{\tilde{\chi}}-k_{ \pm} k_{\|}\right) V(\mathbf{k}, \tilde{\eta}) e^{i \tilde{x} \mu}-\left.\frac{i k_{ \pm}}{\tilde{\chi}} V\left(\mathbf{k}, \eta_{0}\right) e^{i x \mu}\right|_{x=0}+\int_{0}^{\tilde{\chi}} d \chi \frac{\chi}{\tilde{\chi}} i k_{ \pm}\left[\Phi^{\prime}(\mathbf{k}, \eta)-\Psi^{\prime}(\mathbf{k}, \eta)\right] e^{i x \mu}\right) \\
= & \frac{\sqrt{1-\mu^{2}}}{\sqrt{2}}\left[i k V(\mathbf{k}, \tilde{\eta}) e^{i \tilde{x} \mu}+\frac{1+\tilde{z}}{H(\tilde{z})}\left(i k \Psi(\mathbf{k}, \tilde{\eta}) e^{i \tilde{x} \mu}\right.\right. \\
& \left.\left.+\left(-i \frac{k}{\tilde{\chi}}+\mu k^{2}\right) V(\mathbf{k}, \tilde{\eta}) e^{i \tilde{x} \mu}+\left.\frac{i k}{\tilde{\chi}} V\left(\mathbf{k}, \eta_{0}\right) e^{i x \mu}\right|_{x=0}-\int_{0}^{\tilde{\chi}} \frac{d \chi}{\tilde{\chi}} i x\left[\Phi^{\prime}(\mathbf{k}, \eta)-\Psi^{\prime}(\mathbf{k}, \eta)\right] e^{i x \mu}\right)\right],
\end{aligned}
$$

where as before $x=k \chi, \tilde{x}=k \tilde{\chi}$, and $\tilde{\eta}=\eta_{0}-\tilde{\chi}$. Further, we have used that $k_{\|}=\hat{n}^{i} k_{i}=\mu k$, and $k_{ \pm}=m_{\mp}^{i} k_{i}=$ $-\sqrt{1-\mu^{2}} k / \sqrt{2}$. We now apply the spin-lowering operator Eq. (A7) to ${ }_{1} \mathcal{B}$. Note that since ${ }_{ \pm 1} \mathcal{B}(\hat{\mathbf{n}}, \mathbf{k})$ is $\phi$ independent, this is identical to the expression for $ð_{-1} \mathcal{B}$.

$$
\begin{aligned}
\bar{\partial}_{1} \mathcal{B}(\hat{\mathbf{n}}, \mathbf{k})= & \partial_{\mu}\left[\sqrt{1-\mu^{2}}{ }_{1} \mathcal{B}(\hat{\mathbf{n}}, \mathbf{k})\right] \\
= & \frac{{ }_{\mu}}{\sqrt{2}}\left\{( 1 - \mu ^ { 2 } ) \left[i k V(\mathbf{k}, \tilde{\eta}) e^{i \tilde{x} \mu}+\frac{1+\tilde{z}}{H(\tilde{z})}\left(i k \Psi(\mathbf{k}, \tilde{\eta}) e^{i \tilde{x} \mu}+\left(\mu-i \frac{1}{\tilde{x}}\right) k^{2} V(\mathbf{k}, \tilde{\eta}) e^{i \tilde{x} \mu}\right.\right.\right. \\
& \left.\left.\left.+\left.i \frac{1}{\tilde{x}} k^{2} V\left(\mathbf{k}, \eta_{0}\right) e^{i x \mu}\right|_{x=0}-\int_{0}^{\tilde{\chi}} \frac{d \chi}{\tilde{\chi}} i x\left[\Phi^{\prime}(\mathbf{k}, \eta)-\Psi^{\prime}(\mathbf{k}, \eta)\right] e^{i x \mu}\right)\right]\right\} \\
= & \frac{V(\mathbf{k}, \tilde{\eta})}{\sqrt{2} \tilde{\chi}} \hat{Q}_{B S 1}(\tilde{x}) e^{i \tilde{x} \mu}+\frac{1+\tilde{z}}{\sqrt{2} H(\tilde{z})}\left\{\frac{1}{\tilde{\chi}} \Psi(\mathbf{k}, \tilde{\eta}) \hat{Q}_{B S 1}(\tilde{x}) e^{i \tilde{x} \mu}+k^{2} V(\mathbf{k}, \tilde{\eta})\left[\hat{Q}_{B S 2}(\tilde{x})-\frac{1}{\tilde{x}^{2}} \hat{Q}_{B S 1}(\tilde{x})\right] e^{i \tilde{x} \mu}\right. \\
& \left.+\frac{1}{\tilde{\chi}^{2}}\left[\hat{Q}_{B S 1}(x) e^{i x \mu}\right]_{x=0} V\left(\mathbf{k}, \eta_{0}\right)-\int_{0}^{\tilde{\chi}} \frac{d \chi}{\tilde{\chi}} \hat{Q}_{B S 1}(x)\left[\Phi^{\prime}(\mathbf{k}, \eta)-\Psi^{\prime}(\mathbf{k}, \eta)\right] e^{i x \mu}\right\},
\end{aligned}
$$

where we have defined

$$
\begin{aligned}
& \hat{Q}_{B S 1}(x)=-x^{2}-2 x \partial_{x}-x^{2} \partial_{x}^{2} \\
& \hat{Q}_{B S 2}(x)=1+x \partial_{x}+3 \partial_{x}^{2}+x \partial_{x}^{3} .
\end{aligned}
$$

This expression is in the desired form, Eq. (A19), and we see that all coefficients are real. Thus, scalar perturbations only contribute to the (polar) $E$ component of $\mathcal{B}_{i}$.

\section{Tensor modes}

We begin with Eq. (45):

$$
\begin{aligned}
\left({ }_{ \pm 1} \mathcal{B}\right)_{\mathrm{sc}} & =\frac{1+\tilde{z}}{2 H(\tilde{z})} \int_{0}^{\tilde{\chi}} d \chi \frac{\chi}{\tilde{\chi}} \partial_{ \pm} h_{\|}^{\prime} \\
& =\frac{1+\tilde{z}}{2 H(\tilde{z})} \int_{0}^{\tilde{\chi}} d \chi \frac{\chi}{\tilde{\chi}}\left(\left(\partial_{ \pm} h_{k l}^{\prime}\right) \hat{n}^{k} \hat{n}^{l}+\frac{2}{\chi} h_{k l}^{\prime} m_{+}^{k} \hat{n}^{l}\right) .
\end{aligned}
$$

We then decompose $h_{i j}$ into Fourier modes of two polarization states (see Refs. [21,24] for details),

$$
h_{i j}(\mathbf{k}, \eta)=e_{i j}^{+}(\hat{\mathbf{k}}) h^{+}(\mathbf{k}, \eta)+e_{i j}^{\times}(\hat{\mathbf{k}}) h^{\times}(\mathbf{k}, \eta),
$$

where $e_{i j}^{s}(\hat{\mathbf{k}}), s=+, \times$, are transverse (with respect to $\hat{\mathbf{k}}$ ) and traceless polarization tensors normalized through $e_{i j}^{s} e^{s^{\prime} i j}=2 \delta^{s s^{\prime}}$. We assume both polarizations to be independent and to have equal power spectra:

$$
\begin{aligned}
\left\langle h_{s}(\mathbf{k}, \eta) h_{s^{\prime}}\left(\mathbf{k}^{\prime}, \eta^{\prime}\right)\right\rangle= & (2 \pi)^{3} \delta_{D}\left(\mathbf{k}-\mathbf{k}^{\prime}\right) \\
& \times \delta_{s s^{\prime}} \frac{1}{4} T_{T}(k, \eta) T_{T}\left(k, \eta^{\prime}\right) P_{T 0}(k),
\end{aligned}
$$

where $T_{T}(k, \eta)$ is the tensor transfer function, and the primordial tensor power spectrum is denoted as $P_{T 0}(k)$. Further, we can define helicity \pm 2 polarization tensors and Fourier amplitudes through 


$$
e_{i j}^{ \pm 1} \equiv e_{i j}^{+} \pm i e_{i j}^{\times}, \quad h_{\mp 1} \equiv \frac{1}{2}\left(h^{+} \pm i h^{\times}\right) .
$$

Note that $P_{h_{ \pm 1}}(k)=P_{T 0}(k) / 8$. As before, we begin by evaluating the contribution of a single plane wave, assuming that $\mathbf{k}=k \hat{\mathbf{z}}$. We have

$$
\begin{aligned}
e_{ \pm}^{p}(\hat{\mathbf{k}}, \hat{\mathbf{n}}) \equiv e_{i j}^{p}(\hat{\mathbf{k}}) m_{+}^{i}(\hat{\mathbf{n}}) m_{+}^{j}(\hat{\mathbf{n}}) & =\frac{1}{2}(1 \mp p \mu)^{2} e^{i 2 p \phi} \\
e_{\|}^{p}(\hat{\mathbf{k}}) \equiv e_{i j}^{p} \hat{n}^{i} \hat{n}^{j} & =\left(1-\mu^{2}\right) e^{i 2 p \phi} \\
e_{i j}^{p}(\hat{\mathbf{k}}) m_{ \pm}^{i}(\hat{\mathbf{n}}) \hat{n}^{j} & =\frac{\sqrt{1-\mu^{2}}}{\sqrt{2}}(\mu \pm p) e^{i 2 p \phi},
\end{aligned}
$$

where $\mu=\cos \theta$. Restricting to the polarization $p=+1$ first, we have

$$
\begin{aligned}
{ }_{ \pm 1} \mathcal{B}(\mathbf{k}, \hat{\mathbf{n}},+1)= & \frac{1+\tilde{z}}{2 H(\tilde{z})} \int_{0}^{\tilde{\chi}} d \chi \frac{\chi}{\tilde{\chi}}\left\{-\frac{\sqrt{1-\mu^{2}}}{\sqrt{2}}\left(1-\mu^{2}\right) i k\right. \\
& \left.+\frac{\sqrt{2}}{\chi} \sqrt{1-\mu^{2}}(\mu \mp 1)\right\} h_{1}^{\prime}(\mathbf{k}, \eta) e^{i 2 \phi} e^{i x \mu}
\end{aligned}
$$

We now apply the spin-lowering operator Eq. (A7) with $m=2$ to obtain

$$
\begin{aligned}
\overline{\mathrm{d}}_{1} \mathcal{B}(\mathbf{k}, \hat{\mathbf{n}},+1) & =\left[\partial_{\mu}-\frac{2}{1-\mu^{2}}\right] \sqrt{1-\mu^{2}}{ }_{1} \mathcal{B}(\mathbf{k}, \hat{\mathbf{n}},+1) \\
& =\left[\partial_{\mu}-\frac{2}{1-\mu^{2}}\right]\left\{\frac{1+\tilde{z}}{2 H(\tilde{z})} \int_{0}^{\tilde{\chi}} \frac{d \chi}{\tilde{\chi}}\left\{-\frac{\left(1-\mu^{2}\right)^{2}}{\sqrt{2}} i x-\sqrt{2}\left(1-\mu^{2}\right)(1-\mu)\right\} h_{1}^{\prime}(\mathbf{k}, \eta) e^{i 2 \phi} e^{i x \mu}\right\} \\
& \left.=\frac{1+\tilde{z}}{2 H(\tilde{z})} \int_{0}^{\tilde{\chi}} \frac{d \chi}{\tilde{\chi}}\left\{\frac{1}{\sqrt{2}} \hat{Q}_{B T 1}(x)+\sqrt{2} \hat{Q}_{B T 2}(x)\right\}\left(1-\mu^{2}\right) e^{i 2 \phi} e^{i x \mu}\right\} h_{1}^{\prime}(\mathbf{k}, \eta)
\end{aligned}
$$

where

$$
\hat{Q}_{B T 1}(x)=x^{2}+4 x \partial_{x}+x^{2} \partial_{x}^{2}+2 i x, \quad \hat{Q}_{B T 2}(x)=3+x \partial_{x}-i x .
$$

For the other polarization state, we obtain the same result with $\mu \rightarrow-\mu, x \rightarrow-x, \phi \rightarrow-\phi$. Equation (D11) is in the desired form, Eq. (A19). We see that the operators have both real and imaginary parts, signaling that tensor modes contribute to both the polar ("E-mode", through $\operatorname{Re} \hat{Q}_{B T i}$ ) and axial vector (" $B$-mode", through $\operatorname{Im} \hat{Q}_{B T i}$ ).

\section{APPENDIX E: COMPONENTS IN TERMS OF MATTER DENSITY CONTRAST}

This section gives useful expressions for $\mathcal{C},{ }_{ \pm 1} \mathcal{B}, \mathcal{M}$, and the shear in terms of the familiar matter density contrast $\delta_{m}^{\text {sc }}$ in the sc gauge. For convenience we write the velocity $v_{i}$ in terms of a scalar velocity potential $V, v_{i}=V_{, i}$, and relate $V, \Phi, \Psi$ to the density contrast $\delta_{m}^{\text {sc }}$ in the sc gauge through (see Refs. [38,39])

$$
\begin{aligned}
V(\mathbf{k}, \eta) & =a H f k^{-2} D(a(\eta)) \delta_{m}^{\mathrm{sc}}\left(\mathbf{k}, \eta_{0}\right) \\
\Phi(\mathbf{k}, \eta)-\Psi(\mathbf{k}, \eta) & =D_{\Phi_{-}}(k, \eta) \delta_{m}^{\mathrm{sc}}\left(\mathbf{k}, \eta_{0}\right) \\
\Phi(\mathbf{k}, \eta)+\Psi(\mathbf{k}, \eta) & =g(k, \eta) D_{\Phi_{-}}(k, \eta) \delta_{m}^{\mathrm{sc}}\left(\mathbf{k}, \eta_{0}\right) \\
\Rightarrow \Phi(\mathbf{k}, \eta) & =\frac{1}{2}[g+1] D_{\Phi_{-}} \delta_{m}^{\mathrm{sc}}\left(\mathbf{k}, \eta_{0}\right) \\
\Psi(\mathbf{k}, \eta) & =\frac{1}{2}[g-1] D_{\Phi_{-}} \delta_{m}^{\mathrm{sc}}\left(\mathbf{k}, \eta_{0}\right) \\
(\Phi-\Psi)^{\prime}(\mathbf{k}, \eta) & =D_{\mathrm{ISW}}(k, \eta) \delta_{m}^{\mathrm{sc}}\left(\mathbf{k}, \eta_{0}\right),
\end{aligned}
$$

where $f \equiv d \ln D / d \ln a, D(a)$ is the matter growth factor (normalized to unity at $a=1$ ) and we have defined general coefficient functions $D_{\Phi_{-}}, g, D_{\mathrm{ISW}}$ to allow for nonstandard cosmologies. In a $\Lambda$ CDM cosmology (or more generally for a smooth dark energy component), we have

$$
D_{\Phi_{-}}(\mathbf{k}, \eta)=3 \Omega_{m} \frac{a^{2} H^{2}}{k^{2}} D(a(\eta))=3 \Omega_{m 0} \frac{H_{0}^{2}}{k^{2}} a^{-1}(\eta) D(a(\eta)), \quad g(\mathbf{k}, \eta)=0 .
$$

Here, a subscript 0 denotes that the quantity is defined at the present epoch $\eta=\eta_{0}$. We will denote the power spectrum of $\delta_{m}^{\text {sc }}$ at $z=0$ as $P_{m}(k)$, as well as employ $x=k \chi, \tilde{x}=k \tilde{\chi}$ as before. 
We begin with Eq. (42),

\section{Longitudinal scalar}

$$
(\mathcal{C})_{\mathrm{cN}}=-b_{z} \Delta \ln a-\Psi-v_{\|}+\frac{1+\tilde{z}}{H(\tilde{z})}\left(-\partial_{\|} \Psi+\partial_{\|} v_{\|}-v_{\|}^{\prime}+\Phi^{\prime}\right),
$$

where

$$
b_{z}=1-H(\tilde{z}) \partial_{\tilde{z}}\left(\frac{1+\tilde{z}}{H(\tilde{z})}\right)-\frac{\partial \ln r_{0}}{\partial \ln a}, \quad \Delta \ln a=\Psi_{o}-\Psi+v_{\|}-v_{\| o}+\int_{0}^{\tilde{\chi}} d \chi\left[\Phi^{\prime}-\Psi^{\prime}\right]
$$

Note that for a nonevolving ruler in $\Lambda \mathrm{CDM}, b_{z}=3 \Omega_{m}(\tilde{z}) / 2$. For a single plane-wave perturbation, this yields

$$
\begin{aligned}
\mathcal{C}(\mathbf{k}, \hat{\mathbf{n}})= & b_{z}\left\{\left(\Psi(\mathbf{k}, \tilde{\eta})-i \mu \tilde{x} \frac{V(\mathbf{k}, \tilde{\eta})}{\tilde{\chi}}\right) e^{i \tilde{x} \mu}-\left[\left(\Psi\left(\mathbf{k}, \eta_{0}\right)-i \mu x \frac{V\left(\mathbf{k}, \eta_{0}\right)}{\chi}\right) e^{i x \mu}\right]_{x=0}-\int_{0}^{\tilde{\chi}} d \chi\left[\Phi^{\prime}-\Psi^{\prime}\right] e^{i x \mu}\right\} \\
& -\left[\Psi(\mathbf{k}, \tilde{\eta})+i \mu \tilde{x} \frac{V(\mathbf{k}, \tilde{\eta})}{\tilde{\chi}}\right] e^{i \tilde{x} \mu}+\frac{1+\tilde{z}}{H(\tilde{z}) \tilde{\chi}}\left[-i \mu \tilde{x} \Psi(\mathbf{k}, \tilde{\eta})-\mu^{2} \tilde{x}^{2} \frac{V(\mathbf{k}, \tilde{\eta})}{\tilde{\chi}}-i \mu \tilde{x} V^{\prime}(\mathbf{k}, \tilde{\eta})+\tilde{\chi} \Phi^{\prime}(\mathbf{k}, \tilde{\eta})\right] e^{i \tilde{x} \mu} \\
= & \delta_{m}^{\mathrm{sc}}\left(\mathbf{k}, \eta_{0}\right) b_{z}\left\{-\left[\left(\frac{1}{2}(g-1) D_{\Phi_{-}}-\frac{a H f D}{k^{2} \tilde{\chi}} \hat{Q}_{C S 1}(\tilde{x})\right) e^{i x \mu}\right]_{x=0}+\left(\frac{1}{2}(g-1) D_{\Phi_{-}}-\frac{a H f D}{k^{2} \tilde{\chi}} \hat{Q}_{C S 1}(\tilde{x})\right)\right. \\
& \left.-\int_{0}^{\tilde{\chi}} d \chi D_{\mathrm{ISW}} e^{i x \mu}\right\}+\delta_{m}^{\mathrm{sc}}\left(\mathbf{k}, \eta_{0}\right)\left\{-\left[\frac{1}{2}(g-1) D_{\Phi_{-}}+\frac{a H f D}{k^{2} \tilde{\chi}} \hat{Q}_{C S 1}(\tilde{x})\right]+\frac{1+\tilde{z}}{H(\tilde{z}) \tilde{\chi}}\left[-\frac{1}{2}(g-1) D_{\Phi_{-}} \hat{Q}_{C S 1}(\tilde{x})\right.\right. \\
& \left.\left.+\frac{a H f D}{k^{2} \tilde{\chi}} \hat{Q}_{C S 2}(\tilde{x})-(a H f D)^{\prime} \hat{Q}_{C S 1}(\tilde{x})+\frac{\tilde{\chi}}{2}\left(g^{\prime} D_{\Phi_{-}}+(g+1) D_{\mathrm{ISW}}\right)\right]\right\}_{k, \tilde{\eta}} e^{i \tilde{x} \mu},
\end{aligned}
$$

where $\hat{Q}_{C S 1}(x)=x \partial_{x}$, and $\hat{Q}_{C S 2}(x)=x^{2} \partial_{x}^{2}$. This is clearly in the form Eq. (A19), with $r=s=0$, and we can thus immediately apply Eq. (A24):

$$
\begin{aligned}
C_{\mathcal{C}}(l)= & \frac{2}{\pi} \int k^{2} d k P_{m}(k)\left|F_{l}^{\mathcal{C}}(k)\right|^{2} \\
F_{l}^{\mathcal{C}}(k)= & b_{z} F_{l}^{\Delta \ln a}(k)+\left\{-\left[\frac{1}{2}(g-1) D_{\Phi_{-}}+\frac{a H f D}{k^{2} \tilde{\chi}} \hat{Q}_{C S 1}(\tilde{x})\right]+\frac{1+\tilde{z}}{H(\tilde{z}) \tilde{\chi}}\left[-\frac{1}{2}(g-1) D_{\Phi_{-}} \hat{Q}_{C S 1}(\tilde{x})+\frac{a H f D}{k^{2} \tilde{\chi}} \hat{Q}_{C S 2}(\tilde{x})\right.\right. \\
& \left.\left.-(a H f D)^{\prime} \hat{Q}_{C S 1}(\tilde{x})+\frac{\tilde{\chi}}{2}\left(g^{\prime} D_{\Phi_{-}}+(g+1) D_{\mathrm{ISW}}\right)\right]\right\}_{k, \tilde{\eta}} j_{l}(\tilde{x}) \\
F_{l}^{\Delta \ln a}(k) \equiv & \left(\frac{a H f D}{k} \partial_{\tilde{x}}-\frac{1}{2}(g-1) D_{\Phi_{-}}\right)_{\tilde{z}} j_{l}(\tilde{x})+\int_{0}^{\tilde{\chi}} d \chi D_{\mathrm{ISW}} j_{l}(x)
\end{aligned}
$$

\section{Vector}

As derived in Appendix D,

$$
\begin{aligned}
\bar{\partial}_{1} \mathcal{B}(\hat{\mathbf{n}}, \mathbf{k})= & \frac{V(\mathbf{k}, \tilde{\eta})}{\sqrt{2} \tilde{\chi}} \hat{Q}_{B S 1}(\tilde{x}) e^{i \tilde{x} \mu}+\frac{1+\tilde{z}}{\sqrt{2} H(\tilde{z})}\left\{\frac{1}{\tilde{\chi}} \Psi(\mathbf{k}, \tilde{\eta}) \hat{Q}_{B S 1}(\tilde{x}) e^{i \tilde{x} \mu}+k^{2} V(\mathbf{k}, \tilde{\eta})\left[\hat{Q}_{B S 2}(\tilde{x})-\frac{1}{\tilde{x}^{2}} \hat{Q}_{B S 1}(\tilde{x})\right] e^{i \tilde{x} \mu}\right. \\
& \left.+\frac{1}{\tilde{\chi}^{2}}\left[\hat{Q}_{B S 1}(x) e^{i x \mu}\right]_{x=0} V\left(\mathbf{k}, \eta_{0}\right)-\int_{0}^{\tilde{\chi}} \frac{d \chi}{\tilde{\chi}} \hat{Q}_{B S 1}(x)\left[\Phi^{\prime}(\mathbf{k}, \eta)-\Psi^{\prime}(\mathbf{k}, \eta)\right] e^{i x \mu}\right\} \\
= & \delta_{m}^{\mathrm{sc}}\left(\mathbf{k}, \eta_{0}\right)\left\{\left[\frac{a H f D}{\sqrt{2} k^{2} \tilde{\chi}} \hat{Q}_{B S 1}(\tilde{x})+\frac{1+\tilde{z}}{\sqrt{2} H(\tilde{z})}\left(\frac{1}{2 \tilde{\chi}}[g-1] D_{\Phi_{-}}(k, \tilde{a}) \hat{Q}_{B S 1}(\tilde{x})+a H f D\left[\hat{Q}_{B S 2}(\tilde{x})-\frac{1}{\tilde{x}^{2}} \hat{Q}_{B S 1}(\tilde{x})\right]\right)\right]_{\tilde{\eta}} e^{i \tilde{x} \mu}\right. \\
& \left.+\frac{1+\tilde{z}}{\sqrt{2} H(\tilde{z})}\left[(H f D)_{z=0} \frac{1}{\tilde{x}^{2}}\left[\hat{Q}_{B S 1}(x) e^{i x \mu}\right]_{x=0}-\int_{0}^{\tilde{x}} \frac{d \chi}{\tilde{\chi}} D_{\mathrm{ISW}}(k, a(\chi)) \hat{Q}_{B S 1}(x) e^{i x \mu}\right]\right\} .
\end{aligned}
$$

Since ${ }_{ \pm 1} \mathcal{B}$ is a spin-1 quantity, Eq. (A24) with $r=0, s=1$ yields 


$$
\begin{aligned}
C_{\mathcal{B}}^{E E}(l)= & \frac{2}{\pi} \frac{1}{l(l+1)} \int k^{2} d k P_{m}(k)\left|F_{l}^{\mathcal{B E}}(k)\right|^{2} \\
F_{l}^{\mathcal{B E}}(k)= & {\left[\frac{a H f D}{\sqrt{2} k^{2} \tilde{\chi}} \hat{Q}_{B S 1}(\tilde{x})+\frac{1+\tilde{z}}{\sqrt{2} H(\tilde{z})}\left(\frac{1}{2 \tilde{\chi}}[g-1] D_{\Phi_{-}}(k, \tilde{a}) \hat{Q}_{B S 1}(\tilde{x})+a H f D\left[\hat{Q}_{B S 2}(\tilde{x})-\frac{1}{\tilde{x}^{2}} \hat{Q}_{B S 1}(\tilde{x})\right]\right)\right]_{\tilde{\eta}} j_{l}(\tilde{x}) } \\
& +\frac{1+\tilde{z}}{\sqrt{2} H(\tilde{z})}\left[(H f D)_{z=0} \frac{1}{\tilde{x}^{2}}\left[\hat{Q}_{B S 1}(x) j_{l}(x)\right]_{x=0}-\int_{0}^{\tilde{\chi}} \frac{d \chi}{\tilde{\chi}} D_{\mathrm{ISW}}(k, a(\chi)) \hat{Q}_{B S 1}(x) j_{l}(x)\right] .
\end{aligned}
$$

This is the power spectrum of the $E$-mode (polar) component of ${ }_{ \pm} \mathcal{B}$. The operators $\hat{Q}_{B S i}$ as applied to spherical Bessel functions become

$$
\hat{Q}_{B S 1}(x) j_{l}(x)=-l(l+1) j_{l}(x), \quad \hat{Q}_{B S 2}(x) j_{l}(x)=l(l+1) \partial_{x}\left(\frac{j_{l}(x)}{x}\right) .
$$

Note that this implies $\left[\hat{Q}_{B S 2}(x)-x^{-2} \hat{Q}_{B S 1}(x)\right] j_{l}(x)=l(l+1)\left(\partial_{x} j_{l}(x)\right) / x$. Note further that the observer term $\left[\hat{Q}_{B S 1}(x) j_{l}(x)\right]_{x=0}$ is only nonzero for the dipole $l=1$, as expected.

\section{Shear and magnification}

We begin with the shear. Equation (66) evaluated for a plane-wave perturbation yields

$$
\begin{aligned}
{ }_{ \pm 2} \gamma(\hat{\mathbf{n}}, \mathbf{k}) & =-\int_{0}^{\tilde{\chi}} d \chi(\tilde{\chi}-\chi) \frac{\chi}{\tilde{\chi}} \frac{1}{2}\left(1-\mu^{2}\right) k^{2}[\Psi-\Phi]_{\mathbf{k}, \eta} e^{i x \mu} \\
& =\frac{1}{2} \int_{0}^{\tilde{\chi}} d \chi(\tilde{\chi}-\tilde{\chi}) \frac{\chi}{\tilde{\chi}} k^{2} D_{\Phi_{-}}(k, a(\chi))\left(1-\mu^{2}\right) e^{i x \mu} \delta_{m}^{\mathrm{sc}}\left(\mathbf{k}, \eta_{0}\right) .
\end{aligned}
$$

In order to obtain a scalar quantity, we now apply Eq. (A8) with $m=0$, yielding

$$
\begin{aligned}
\bar{\partial}_{2}^{2} \gamma(\hat{\mathbf{n}}, \mathbf{k}) & =\partial_{\mu}^{2}\left[\left(1-\mu^{2}\right)_{2} \gamma\right]=\frac{1}{2} \int_{0}^{\tilde{\chi}} d \chi(\tilde{\chi}-\chi) \frac{\chi}{\tilde{\chi}} k^{2} D_{\Phi_{-}}(k, a(\chi)) \partial_{\mu}^{2}\left[\left(1-\mu^{2}\right)^{2} e^{i x \mu}\right] \delta_{m}^{\mathrm{sc}}\left(\mathbf{k}, \eta_{0}\right) \\
& =\frac{1}{2} \int_{0}^{\tilde{\chi}} d \chi(\tilde{\chi}-\chi) \frac{\chi}{\tilde{\chi}} k^{2} D_{\Phi_{-}}(k, a(\chi)) \hat{Q}_{\gamma S}(x) e^{i x \mu} \delta_{m}^{\mathrm{sc}}\left(\mathbf{k}, \eta_{0}\right),
\end{aligned}
$$

where we have defined

$$
\hat{Q}_{\gamma S}(x)=4+x^{2}+8 x \partial_{x}+\left(12+2 x^{2}\right) \partial_{x}^{2}+8 x \partial_{x}^{3}+x^{2} \partial_{x}^{4}
$$

This is again in the desired form Eq. (A19), and applying Eq. (A24) with $r=0, s=2$ yields the angular power spectrum of the shear $E$ modes,

$$
C_{\gamma}^{E E}(l)=\frac{2}{\pi} \frac{(l-2) !}{(l+2) !} \int k^{2} d k P_{m}(k)\left|F_{l}^{\gamma E}(k)\right|^{2}, \quad F_{l}^{\gamma E}(k)=\frac{1}{2} \int_{0}^{\tilde{\chi}} d \chi(\tilde{\chi}-\chi) \frac{\chi}{\tilde{\chi}} k^{2} D_{\Phi_{-}}(k, a(\chi)) \hat{Q}_{\gamma S}(x) j_{l}(x) .
$$

The operator $\hat{Q}_{\gamma S}$ applied to spherical Bessel functions is

$$
\hat{Q}_{\gamma S}(x) j_{l}(x)=\frac{(l+2) !}{(l-2) !} \frac{j_{l}(x)}{x^{2}} .
$$

The magnification contains a number of terms, which are evaluated in Appendix B of Ref. [24]. Here we only repeat the final result:

$$
\begin{aligned}
C_{\mathcal{M}}(l) & =\frac{2}{\pi} \int k^{2} d k P_{m}(k)\left|F_{l}^{\mathcal{M}}(k)\right|^{2} \\
F_{l}^{\mathcal{M}}(k) & =2\left[\frac{1}{a H \tilde{\chi}}-1\right] F_{l}^{\Delta \ln a}(k)-\left([g+1] D_{\Phi_{-}}\right)_{\tilde{z}} j_{l}(\tilde{x})+l(l+1) \int_{0}^{\tilde{\chi}} d \chi \frac{\tilde{\chi}-\chi}{\chi \tilde{\chi}} D_{\Phi_{-}} j_{l}(x)+2 \int_{0}^{\tilde{\chi}} \frac{d \chi}{\tilde{\chi}} D_{\Phi_{-}} j_{l}(x),
\end{aligned}
$$

where $F_{l}^{\Delta \ln a}(k)$ is defined in Eq. (E6). 
[1] A. G. Riess, A. V. Filippenko, P. Challis, A. Clocchiatti, A. Diercks, P. M. Garnavich, R. L. Gilliland, C. J. Hogan, S. Jha, R. P. Kirshner et al., Astron. J. 116, 1009 (1998).

[2] S. Perlmutter, S. Gabi, G. Goldhaber, A. Goobar, D. E. Groom, I. M. Hook, A. G. Kim, M. Y. Kim, J. C. Lee, R. Pain et al., Astrophys. J. 483, 565 (1997).

[3] D. J. Eisenstein, I. Zehavi, D. W. Hogg, R. Scoccimarro, M. R. Blanton, R. C. Nichol, R. Scranton, H.-J. Seo, M. Tegmark, Z. Zheng et al., Astrophys. J. 633, 560 (2005).

[4] S. Cole, W. J. Percival, J. A. Peacock, P. Norberg, C. M. Baugh, C.S. Frenk, I. Baldry, J. Bland-Hawthorn, T. Bridges, R. Cannon et al., Mon. Not. R. Astron. Soc. 362, 505 (2005).

[5] A. Lewis and A. Challinor, Phys. Rep. 429, 1 (2006).

[6] O. Zahn and M. Zaldarriaga, Astrophys. J. 653, 922 (2006).

[7] T. Lu and U.-L. Pen, Mon. Not. R. Astron. Soc. 388, 1819 (2008).

[8] J. Yoo, A. L. Fitzpatrick, and M. Zaldarriaga, Phys. Rev. D 80, 083514 (2009).

[9] A. Challinor and A. Lewis, Phys. Rev. D 84, 043516 (2011).

[10] C. Bonvin and R. Durrer, Phys. Rev. D 84, 063505 (2011).

[11] D. Jeong, F. Schmidt, and C. M. Hirata, Phys. Rev. D 85, 023504 (2012).

[12] R. Sachs, Proc. R. Soc. A 264, 309 (1961).

[13] M. Sasaki, Mon. Not. R. Astron. Soc. 228, 653 (1987).

[14] F. Bernardeau, C. Bonvin, and F. Vernizzi, Phys. Rev. D 81, 083002 (2010).

[15] C. Pitrou, J.-P. Uzan, and T. S. Pereira, arXiv:1203.6029.

[16] S. Dodelson, E. Rozo, and A. Stebbins, Phys. Rev. Lett. 91, 021301 (2003).

[17] P. Catelan, M. Kamionkowski, and R. D. Blandford, Mon. Not. R. Astron. Soc. 320, L7 (2001).

[18] U.-L. Pen, R. Sheth, J. Harnois-Deraps, X. Chen, and Z. Li, arXiv:1202.5804.
[19] D. Jeong and M. Kamionkowski, Phys. Rev. Lett. 108, 251301 (2012).

[20] S. Dodelson, Phys. Rev. D 82, 023522 (2010).

[21] F. Schmidt and D. Jeong, arXiv:1205.1514.

[22] K. W. Masui and U.-L. Pen, Phys. Rev. Lett. 105, 161302 (2010).

[23] L. Book, M. Kamionkowski, and F. Schmidt, Phys. Rev. Lett. 108, 211301 (2012).

[24] D. Jeong and F. Schmidt, arXiv:1205.1512.

[25] V. Gluscevic, M. Kamionkowski, and A. Cooray, Phys. Rev. D 80, 023510 (2009).

[26] N. Kaiser, Mon. Not. R. Astron. Soc. 227, 1 (1987).

[27] L. Hui and P. B. Greene, Phys. Rev. D 73, 123526 (2006).

[28] C. Bonvin, R. Durrer, and M. A. Gasparini, Phys. Rev. D 73, 023523 (2006).

[29] F. Schmidt, A. Leauthaud, R. Massey, J. Rhodes, M. R. George, A.M. Koekemoer, A. Finoguenov, and M. Tanaka, Astrophys. J. Lett. 744, L22 (2012).

[30] E. Fermi, Atti Acad. Naz. Lincei Rend. Cl. Sci. Fis. Mat. Nat. 31, 21 (1922).

[31] F. K. Manasse and C. W. Misner, J. Math. Phys. (N.Y.) 4, 735 (1963).

[32] W. Hu, Phys. Rev. D 62, 043007 (2000).

[33] D. Jeong, F. Schmidt, and E. Sefusatti, Phys. Rev. D 83, 123005 (2011).

[34] J. N. Goldberg, A. J. Macfarlane, E. T. Newman, F. Rohrlich, and E. C. G. Sudarshan, J. Math. Phys. (N.Y.) 8, 2155 (1967).

[35] M. Zaldarriaga and U. Seljak, Phys. Rev. D 55, 1830 (1997).

[36] S. Weinberg, Cosmology (Oxford University Press, New York, 2008).

[37] T. Pyne and M. Birkinshaw, Astron. J. 415, 459 (1993).

[38] F. Schmidt, Phys. Rev. D 78, 043002 (2008).

[39] W. Hu and I. Sawicki, Phys. Rev. D 76, 104043 (2007). 